\title{
Double Cones and the Diverse Connectivity of Photoreceptors and Bipolar Cells in an Avian Retina
}

\author{
Anja Günther, ${ }^{1}{ }^{\circledR}$ Karin Dedek, ${ }^{1,2}$ Silke Haverkamp, ${ }^{3}$ Stephan Irsen, ${ }^{4}$ Kevin L. Briggman, ${ }^{3}$ and \\ ${ }^{(-)}$Henrik Mouritsen ${ }^{1,2}$ \\ ${ }^{1}$ Neurosensorics/Animal Navigation, Institute for Biology and Environmental Sciences, Carl von Ossietzky University of Oldenburg, 26129 \\ Oldenburg, Germany, ${ }^{2}$ Research Center for Neurosensory Sciences, Carl von Ossietzky University of Oldenburg, 26129 Oldenburg, Germany, \\ ${ }^{3}$ Department of Computational Neuroethology, Center of Advanced European Studies and Reasearch (caesar), 53175 Bonn, Germany, and ${ }^{4}$ Electron \\ Microscopy and Analytics, Center of Advanced European Studies and Research (caesar), 53175 Bonn, Germany
}

Double cones are the most common photoreceptor cell type in most avian retinas, but their precise functions remain a mystery. Among their suggested functions are luminance detection, polarized light detection, and light-dependent, radical pairbased magnetoreception. To better understand the function of double cones, it will be crucial to know how they are connected to the neural network in the avian retina. Here we use serial sectioning, multibeam scanning electron microscopy to investigate double-cone anatomy and connectivity with a particular focus on their contacts to other photoreceptor and bipolar cells in the chicken retina. We found that double cones are highly connected to neighboring double cones and with other photoreceptor cells through telodendria-to-terminal and telodendria-to-telodendria contacts. We also identified 15 bipolar cell types based on their axonal stratifications, photoreceptor contact pattern, soma position, and dendritic and axonal field mosaics. Thirteen of these 15 bipolar cell types contacted at least one or both members of the double cone. All bipolar cells were bistratified or multistratified. We also identified surprising contacts between other cone types and between rods and cones. Our data indicate a much more complex connectivity network in the outer plexiform layer of the avian retina than originally expected.

Key words: bipolar cell types; connectivity; double cones; photoreceptors; serial section multibeam scanning electron microscopy

\section{Significance Statement}

Like in humans, vision is one of the most important senses for birds. Here, we present the first serial section multibeam scanning electron microscopy dataset from any bird retina. We identified many previously undescribed rod-to-cone and cone-tocone connections. Surprisingly, of the 15 bipolar cell types we identified, 11 received input from rods and 13 of 15 received at least part of their input from double cones. Therefore, double cones seem to play many different and important roles in avian retinal processing, and the neural network and thus information processing in the outer retina are much more complex than previously expected. These fundamental findings will be very important for several fields of science, including vertebrate vision, avian magnetoreception, and comparative neuroanatomy.

Received Sep. 23, 2020; revised Mar. 3, 2021; accepted Apr. 1, 2021.

Author contributions: A.G., K.D., S.H., S.I., K.L.B., and H.M. designed research; A.G., S.H., and S.I. performed research; K.L.B. contributed unpublished reagents/analytic tools; A.G., K.D., S.H., and H.M. analyzed data; A.G., K.D., S.H., K.L.B., and H.M. wrote the paper.

This project was funded by the Air Force Office of Scientific Research (Air Force Materiel Command USAF award FA9550-14-1-0095) to H.M., by the Deutsche Forschungsgemeinschaft (DFG; Grant SFB1372: Navigation and Magnetoreception in Vertebrates, Project 395940726 and DFG Research Training Group Grant GRK 1885; both to H.M. and K.D.). H.M. also received funding from the European Research Council [under the European Union's Horizon 2020 research and innovation program, Grant 810002 (Synergy Grant: "QuantumBirds")]. In addition, funding was provided by Stiftung Caesar (to S.H., S.I., and K.L.B.). We thank Pranav Seth for volunteering to help grouping the bipolar cells. We also thank Kara Fulton and Timothy Lee for support in performing additional analyses using MATLAB.

The authors declare no competing financial interests.

Correspondence should be addressed to Henrik Mouritsen at henrik.mouritsen@uni-oldenburg.de.

https://doi.org/10.1523/JNEUROSCI.2495-20.2021

Copyright $\odot 2021$ the authors

\section{Introduction}

The visual system of birds is one of the most complex among vertebrates, and its importance for birds is reflected by the size of their eyes occupying up to $50 \%$ of the cranial volume (Waldvogel, 1990), their large number of retinal neurons (Seifert et al., 2020), and the presence of one or two foveas and/or a visual streak in the retina of many avian species (Wood, 1917). It is thus surprising that little is known about the anatomic ultrastructure and the connectivity of avian retinal cells.

Beside rods, birds have four types of single cones enabling tetrachromatic vision (Hart, 2001). Additionally, birds have double cones, which often make up $40 \%$ of the cone population in the retina. Double cones are common among vertebrates except for 
eutherian mammals, elasmobranchs, and catfish (Walls, 1942; Ebrey and Koutalos, 2001). In contrast to some fish double cones, both members of bird double cones contain the same long wavelength-sensitive opsin (LWS; Hart, 2001). The principal member (PR) contains one oil droplet, whereas the accessory member (AC) can have several oil droplets that can differ in size and number (Pedler and Boyle, 1969). In general, oil droplets act as long-pass filters and can have different absorption properties (Stavenga and Wilts, 2014).

The function of double cones is still debated (Seifert et al., 2020) and may differ between vertebrate classes. In fish, double cones have been implicated in color vision because of different opsins in the individual double-cone members (Ebrey and Koutalos, 2001). Additionally, they might be involved in polarization vision since they form mosaics with neighboring double cones being oriented $90^{\circ}$ to each other (Horváth, 2014). Double cones in birds could play a role in movement detection, because the spectral sensitivity of motion-sensitive cells is comparable to the spectral sensitivity of the LWS pigments of birds expressed in double cones and long-wavelength single cones (Jones and Osorio, 2004; Osorio and Vorobyev, 2005). Furthermore, behavioral conditioning experiments link double cones to mediating fine pattern recognition (Lind and Kelber, 2011).

Additionally, double cones were suggested to be involved in light-dependent, radical pair-based magnetoreception (Zapka et al., 2009; Hore and Mouritsen, 2016; Günther et al., 2018), which is important for orientation and navigation (Wiltschko and Wiltschko, 1995; Kishkinev et al., 2015; Chernetsov et al., 2017; Mouritsen, 2018). Double cones of several bird species including the chicken express cryptochrome 4 (Günther et al., 2018), which could be the primary magnetic sensory molecule as it binds the crucial FAD (flavin adenine dinucleotide) cofactor (Hore and Mouritsen, 2016; Zoltowski et al., 2019), and is magnetically sensitive (Xu et al., 2021). Double cones could be suitable locations for magnetoreceptive molecules since light intensity and polarization information could be separated from the magnetic field information by having two sensors oriented differently in each member of double cones (Worster et al., 2017).

Fundamental anatomic insights into pigeon photoreceptors were provided by Mariani and Leure-du Pree (1978), showing, for example, that rods and double cones stratify in the first (outermost) stratum of the tristratified outer plexiform layer (OPL), which was also found in chicken (Tanabe et al., 2006). Furthermore, electron microscopic data from chick retina suggest a coupling of both members of a double cone through junction-like structures (Nishimura et al., 1981). However, a systematic structural analysis of double cones is missing. Two studies identified bipolar cell types in the chicken retina (Quesada et al., 1988) and pigeon retina (Mariani, 1987). Although these studies provide important knowledge about photoreceptors and bipolar cells in the avian retina, information on potential photoreceptor/photoreceptor and bipolar cell/photoreceptor connections are lacking.

Since these classic electron microscopic studies, electron microscopic techniques underwent a constant development (for review, see Peddie and Collinson, 2014). By using a new development, namely serial sectioning multibeam scanning electron microscopy (ssmSEM; Eberle and Zeidler, 2018), the aim of the present study was to provide a detailed analysis of the anatomy of double cones and their connections to other photoreceptor and bipolar cell types. Furthermore, we reconstructed 74 complete bipolar cells through which an extended bipolar cell type classification emerged.

\section{Materials and Methods}

Animals. One-week-old domestic chickens (Gallus gallus domesticus) were hatched in the teaching and research station Frankenforst (University of Bonn, Koenigswinter, Germany). All animal procedures were approved by the Animal Care and Use Committees of the Niedersächsisches Landesamt für Verbraucherschutz und Lebensmittelsicherheit (LAVES) and the Landesamt für Natur, Umwelt und Verbraucherschutz NordrheinWestfalen (LANUV). The age of an experimental animal may have a crucial influence on the morphology and connectivity of the analyzed cells. However, retinal cell type differentiation and synaptogenesis in the plexiform layers in chicken already starts at an embryonic stage (Drenhaus et al., 2003, 2007), and major receptor types can already be detected at embryonic day 12 (Hering and Kröger, 1996; for review, see Mey and Thanos, 2000). Therefore, the cell development in our 1-week-old chicken is completed and the synaptogenesis is advanced, but it is unclear whether it is entirely completed.

Sample preparation for electron microscopic recordings. Birds were killed by decapitation, and eyes were removed immediately. Lens apparatus and vitreous body were removed, and eyecups were fixed in a 0.08 M cacodylate (Sigma-Aldrich) buffer, $\mathrm{pH} 7.4$, containing $2.5 \%$ paraformaldehyde (PFA; Carl Roth) and $1.25 \%$ glutaraldehyde (Electron Microscopy Sciences) for $30 \mathrm{~min}$ at room temperature. Retinas were removed from the eyecup and transferred into a $0.08 \mathrm{M}$ cacodylate buffer, pH 7.4, two times for 15 min each.

Basic staining procedure was performed after the technique in the study by Hua et al. (2015). Before embedding, the tissue was dehydrated through a graded ethanol series $(50 \%, 75 \%$, and $100 \%, 30$ min each at $4^{\circ} \mathrm{C}$ ), followed by washing three times in $100 \%$ anhydrous acetone (VWR) at room temperature for $30 \mathrm{~min}$ each. For Epon infiltration, the tissue was first incubated in 1:2 mixture of anhydrous acetone and Embed812 resin hard formulation (Embed812, $20 \mathrm{ml}$; dodecenyl succinic anhydride, $9 \mathrm{ml}$; $N$-methylaniline, $12 \mathrm{ml}$; DMP-30, $0.72 \mathrm{ml}$; Electron Microscopy Sciences) overnight at room temperature followed by $8 \mathrm{~h}$ incubation in pure Embed 812 resin at room temperature. Retinas were cut into smaller pieces, and the position within the retina was mapped before transferring the smaller tissue pieces into embedding molds (Ted Pella) for polymerization at $70^{\circ} \mathrm{C}$ for $48 \mathrm{~h}$.

Sample sectioning and data acquisition for electron microscopy. After polymerization, a piece of the dorsal periphery in the left eye was pretrimmed with a hand saw and afterward trimmed to a block face of $\sim 900 \times 250 \mu \mathrm{m}$ using an ultramicrotome (model UC7, Leica). For the $3 \mathrm{D}$ reconstruction dataset, serial sectioning of $40-\mathrm{nm}$-thick slices from a sample in the periphery of the left eye of one chicken was performed with an Ultra ATS Diamond Knife (DiAtome) with a knife angle of $35^{\circ}$ (Science Services). A total of 271 sections $900 \times 250 \mu \mathrm{m}$ in size were collected on a glow discharge silicon wafer (Active Bizz) and dried on a heating plate at $50^{\circ} \mathrm{C}$ until the water was fully evaporated. The wafer was mounted with silver paint (Plano) on a MultiSEM microscope (Zeiss) universal holder version 2 and stored in a heated vacuum chamber until further use. An overview image from the complete wafer was recorded with a Axio Imager.A2 Vario Microscope (Zeiss). Individual slices were marked and tracked using Zen2 (blue edition; Zeiss). The chicken sample was recorded with a 91-parallel-beam MultiSEM 506 Microscope (Zeiss), where the parallel beams are hexagonally arranged to decrease electron-optical aberrations (Eberle et al., 2015). Recordings were performed with a beam current of $591 \mathrm{pA}$, a landing energy of $1.5 \mathrm{keV}$ (kilo-electron volt), $400 \mathrm{~ns}$ pixel dwell time per beam, and a pixel size of $4 \mathrm{~nm}$. A volume area of $\sim 9 \times 10^{3} \mu \mathrm{m}^{3}$ was imaged, resulting in 11,985 hexagonally shaped multibeam fields of view ( $\mathrm{mFOVs}$ ), containing more than 1 million high-resolution images and $\sim 1.36 \times 10^{10}$ megapixels. Hexagonal mFOVs consisted of 91 rectangular, single-beam image tiles with a tile size of $3800 \times 3292$ pixels for each tile. Tile overlap was $500 \mathrm{~nm}$, and $\mathrm{mFOV}$ overlap was $8 \%$. Single-beam images were stored as .tif files accumulating 14.3 TB of storage space. To correct for scan distortion, a built-in algorithm was used. 2D alignment of all tiles per section was also performed by a built-in algorithm. In total, 271 sections were recorded.

For detailed 2D analysis of double cone morphological structures, 40-nm-thick sections of nine chickens were cut with an Ultra Diamond 
Knife with a $35^{\circ}$ knife angle (DiAtome) and transferred on carboncoated copper grids with a hole size of $35 / 10 \mathrm{~nm}$ (model S35/10, Quantifoil). Images were recorded with a analytical electron microscope (model JEM-2200FS, Jeol) at an energy of $200 \mathrm{keV}$ with a CMOS (complementary metal-oxide-semiconductor) camera (model TEM-CAM F416, TVIPS) at a magnification of $40,000 \times$, resulting in a pixel size of $0.322 \mathrm{~nm}$

Image processing and reconstruction of electron microscopic serial sections. Because of the tile overlap of $500 \mathrm{~nm}$ of the mFOVs, the overlapping part of the image was exposed twice to an electron beam, which caused higher bleaching than in the rest of the image. To correct for these differences in brightness, a self-written script containing a bandpass filter was applied on every single tile. Image processing was performed using the plugin TrakEM2 from the Fiji package (Schindelin et al., 2012). All single tiles from one section were loaded into TrakEM2 using the provided text file with individual tile coordinates. To decrease the size of the dataset and increase processing speed, the dataset was divided into seven subvolumes. For each subvolume, a single image per section was created. Single images were afterward merged into one 3D dataset using the tif $2 \mathrm{mrc}$ program from the imod package (Kremer et al., 1996). For 3D alignment of the individual sections in the subvolumes, we used the MIDAS program from the imod package. For the 3D volume reconstruction of one individual double cone, the alignment of a smaller subvolume reaching from the pigment epithelium to the beginning of the inner nuclear layer (INL) and a size of $47.7 \times 75.6 \mu \mathrm{m} \times$ $10.4 \mu \mathrm{m}$ was refined using MIDAS. Because of memory limitation on the computer, we created a volume of $140 \times 148 \times 10.4 \mu \mathrm{m}$ size reaching from the outer nuclear layer (ONL) to the ganglion cell layer (GCL) to reconstruct complete bipolar cells and photoreceptor cells. For all 3D volume reconstructions of individual cells, the $3 \mathrm{dmod}$ program from imod was used (Extended Data Fig. 1-1F, the two subvolumes analyzed).

Analysis of photoreceptor cells and bipolar cells in a $3 D$ volume. To calculate the ratio of the different photoreceptor cell types, photoreceptors of the complete dataset were analyzed. Only photoreceptor cells that could be clearly identified as one specific type of photoreceptor went into the statistical analysis. Based on the stratification level in the OPL, long-wavelength single cones (red cones) and middle-wavelength single cones (green cones) could not be distinguished and were sampled in one group. Likewise, short-wavelength single cones (blue cones) and ultrashort-wavelength single cones (violet cones) could not be distinguished and were also sampled in one group. To further analyze the structure and connectivity strength of the terminals of the cells, we counted all the ribbon synapses present in 20 terminals of rods, single cones, and double cones. Afterward, the mean and SD were calculated. Telodendria or bipolar cell dendrites that reached into the terminal of a photoreceptor and ended there with contacting the terminal other than at a ribbon synapse were classified as "basal contact." Telodendria or bipolar cell dendrites that reached into the terminal of a photoreceptor and ended without making a contact were classified as "no clear contact." If a bipolar cell dendrite or photoreceptor telodendrion contacted a terminal from a photoreceptor but did not end there, it was also classified as no clear contact. For evaluating the dendritic and axonal fields of the bipolar cells, we drew the convex hull for each axon terminal and dendritic field. Classification of bipolar cell types was performed blindly by four untrained researchers to avoid bias. Connectivity matrices were calculated based on the normalized number of basal or ribbon synapses, respectively, using MATLAB (Higham and Higham, 2016). Presynaptic and postsynaptic distributions from basal and ribbon contacts were calculated as follows: for the presynaptic distribution of basal contacts, we counted the basal contacts (Extended Data Fig. 3-2, triangles) within the terminal of one photoreceptor cell type to a specific postsynaptic cell [Extended Data Fig. 3-2A; e.g., bright blue for accessory member (AC)] and divided it by the number of all the detected basal contacts in the terminals (Extended Data Fig. 3-2A, all triangles). The same procedure was performed for the presynaptic distribution of ribbon contacts, where we divided the number of ribbon contacts from one cell type by the number of all ribbon synapses identified within the terminal. For the postsynaptic distribution of synapses, we also counted all the basal and ribbon contacts that a postsynaptic cell type made within the terminal of a specific photoreceptor cell type (Extended Data Fig. 3-2A, triangles or squares in bright blue for $\mathrm{AC}$ ) and divided it by the total number of basal or ribbon synaptic contacts that photoreceptor cell type made (not shown in the figure).

Basal contacts were divided into three classes based on their distance to the nearest ribbon synaptic contact: triad associated, middle non-triad associated, and marginal non-triad associated (Extended Data Figs. 4-1, 4-2, 4-3, 4-4, 4-5, 4-6, 4-7, 4-8, 4-9, 4-10, 4-11, 4-12, 4-13, 4-14, 4-15). Basal contacts were classified as middle non-triad associated if at least two dendrites separated these dendrites from the nearest ribbon synapse and as marginal non-triad associated if they contacted the terminal at the outer margin or at elongations of the terminal other than telodendria (Tsukamoto and Omi, 2015).

The stratification profiles seen in Figure 5 for each bipolar cell type were calculated as probability density function estimates, using the ScatterHist function in MATLAB after calculating the mean cell volume of each bipolar cell type for each drawn contour. In Extended Data Figs. $4-1,4-2,4-3,4-4,4-5,4-6,4-7,4-8,4-9,4-10,4-11,4-12,4-13,4-14$, $4-15 B$, the sum of each cell volume along the $y$-axis was calculated and overlayed with the total sum of all cells within the volume (black line).

Immunohistochemistry. For immunohistochemical analysis, eyecups from four 1-week-old chickens were prepared by cornea dissection followed by removal of the lens and vitreous body. Eyecups were fixed in $4 \%$ PFA/PBS for $30 \mathrm{~min}$ and afterward washed three times for $10 \mathrm{~min}$ in PBS. Part of the retina was removed from the eyecup as stripes (width, $\sim 4 \mathrm{~mm}$ ), reaching from the dorsal periphery to the center tip of the pecten. The retinal stripes were afterward embedded in $4 \%$ high-melting agarose. For vibratome sections, $100-\mu \mathrm{m}$-thick vibratome sections were collected, and triple staining using GNB3 antibody (catalog \#LS-B8347, Lifespan Biosciences), diluted 1:200; PKC $\alpha$ clone MC5 (catalog \#K01107M, Biodesign International), diluted 1:100; and Ctbp2 (catalog \#193003, Synaptic Systems), diluted 1:5000 in PBS including 1\% Triton $\mathrm{X}-100$ and $3 \%$ normal donkey serum, was performed overnight at room temperature. On the next day, retina slices were washed four times for 10 min in PBS, and GNB3, PKC, and Ctbp2 visualization was performed using Alexa Fluor 488, Cy3, and Alexa Fluor 647-conjugated secondary antibodies (Dianova) 1:500, in PBS including 1\% Triton X-100 and 3\% normal donkey serum for $2 \mathrm{~h}$ at room temperature. In addition to the secondary antibodies, we included DAPI (1:10,000; Sigma-Aldrich) to stain cell nuclei. Retinal slices were again washed four times for $10 \mathrm{~min}$, mounted on slides, and covered with Aqua-Poly/Mount (Polysciences) and a coverslip. Confocal micrographs of fluorescent retinas were analyzed with a TCS SP8 Confocal Microscope (Leica Camera) using the 405, 488, 554 and $647 \mathrm{~nm}$ lines and the PMT (photomultiplier) settings) settings were chosen to avoid cross talk between the different lines. Scanning was performed with the glycerol immersion HC PL APO $63 \times 1$ 1.30 GLYC CORR CS2 at a resolution of $2048 \times 2048$ pixels. Stacks were recorded with a thickness of $12 \mu \mathrm{m}$, and final images were $z$-projections of the complete stack adjusted in brightness and contrast in ImageJ (NIH).

\section{Results}

\section{Identification of different photoreceptor types}

We used the novel ssmSEM technique to acquire a high-resolution $3 \mathrm{D}$ electron microscopic dataset from a $900 \times 250 \times 10.4$ $\mu \mathrm{m}$ area in the dorsal periphery of the left retina of a 1-week-old chicken to analyze the morphology of double cones in 3D and gain insights into their connections to bipolar cells and other photoreceptor cells. For a first estimation, we identified all the photoreceptor cell types in the complete dataset using already described morphologic features, such as the presence of an oil droplet in all cones (Bowmaker, 2008), the location of the cell bodies of photoreceptor cells in the ONL, the ending of the synaptic terminals in the three strata of the OPL (Mariani and Leure-du Pree, 1978; Mariani, 1987), and glycogen accumulations in the inner segments of rods (hyperboloid) and the accessory member of the double cones (paraboloid; Meyer and May, 1973). As the green and red single cones both have their synaptic 
A

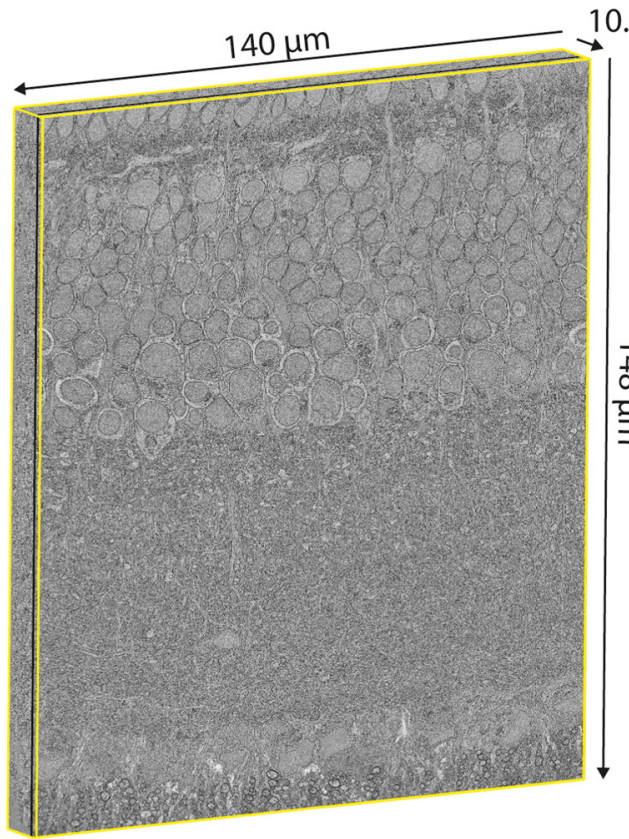

B

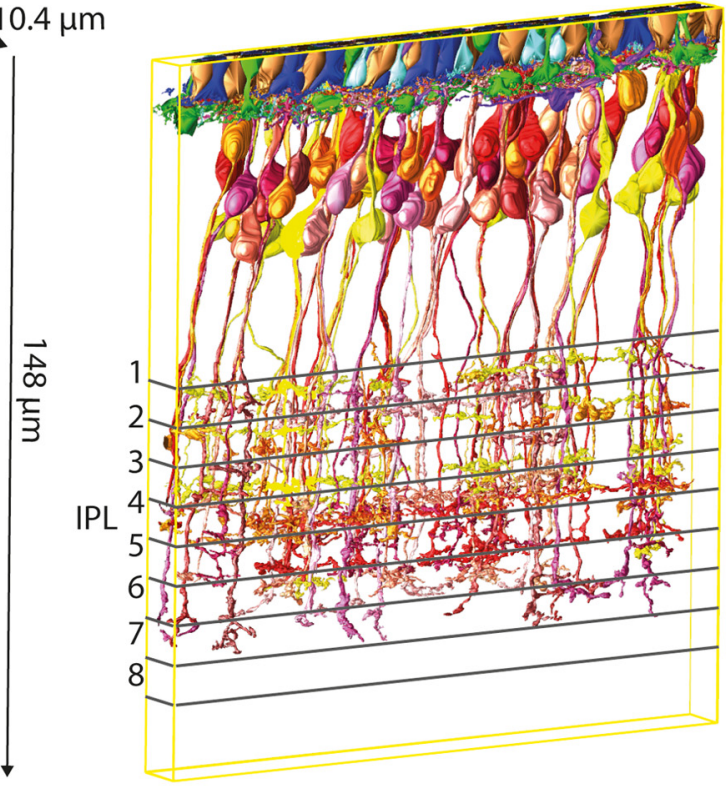

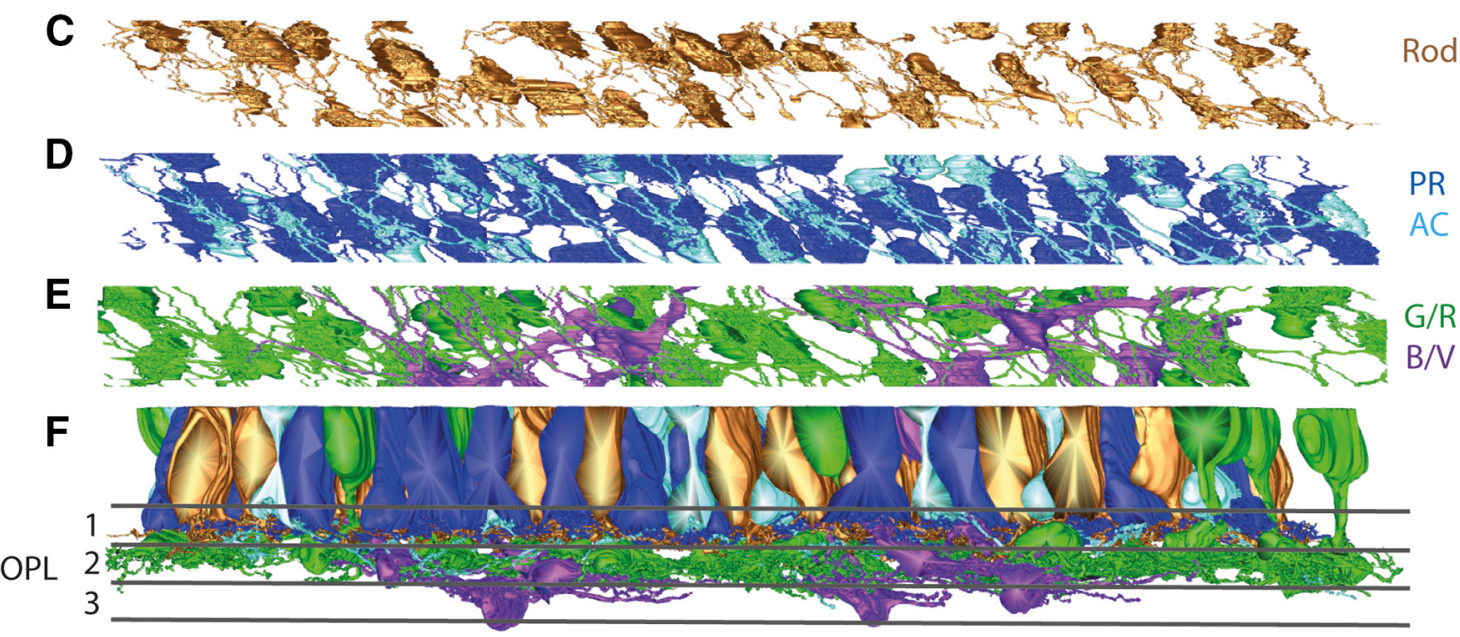

Figure 1. 3D reconstructed subvolume used for bipolar and photoreceptor cell connectivity analysis. $A, 3 \mathrm{D}$ volume representation of the original electron microscopy (EM) dataset highlighting the dimensions of the dataset. $\boldsymbol{B}$, Model representation of all double cones (principal member in dark blue and accessory member in bright blue), rods (brown), green/red cones (green), and blue/violet single cones (magenta), as well as all complete bipolar cells grouped in the identified bipolar cell types indicated in different shades of yellow-orange-red-violet. $\boldsymbol{C}$, Bottom view of double cone (principal member in dark blue and accessory member in bright blue) terminals. $\boldsymbol{D}$, Bottom view of green/red cone (green) and blue/violet cone (magenta) terminals. $\boldsymbol{E}$, Bottom view of rod terminals (brown). $\boldsymbol{F}$, Front view of all photoreceptor cell types within the volume with OPL lamina highlighted. Examples for the trackability of the dataset are given in Extended Data Figure 1-1, including an outline of a complete section with the highlighted subvolumes.

terminals located in the second strata and the blue and violet single cones both have their synaptic terminals located in the third strata in the OPL (Mariani and Leure-du Pree, 1978), we were not able to differentiate between the two cell types of each pair and therefore grouped them together as green/red (G/R) and blue/violet $(\mathrm{B} / \mathrm{V})$ cones. In total, 706 photoreceptor cells were counted. Two hundred twenty-eight of these were identified as double cones, 130 as combined green (medium-wavelength sensitive) or red (LWS) single cones, 51 as blue [short-wavelength sensitive (SWS) 2)] or violet (SWS1) single cones, and 244 as rods. We were able to identify 53 additional photoreceptors as single cones, but we could not characterize them further because essential morphologic features were missing (e.g., the terminal within the OPL). In summary, we found $32.3 \%$ double cones, $34.6 \%$ rods, and $33.1 \%$ single cones. Thus, $49.4 \%$ of all cones were double cones.
In the process of collecting serial sections, some slices were lost, leading to a $\sim 160 \mathrm{~nm}$ gap (Fig. $1 \mathrm{~A}$, black line) in the $140 \times 148 \times 10.4 \mu \mathrm{m}$ subvolume. Despite this gap, we were still able to track most of the processes from photoreceptors as well as the dendrites and axons from bipolar cells (Fig. 1B, Extended Data Fig. 1-1).

The terminals of the rods and both members of double cones form a mosaic (Fig. 1C,D), as do the terminals from the green/red cones (Fig. 1E, green). The terminals of the blue/violet cones seem to cluster together (Fig. 1E, magenta). The photoreceptor terminals from rods and double cones can be found in stratum 1 of the OPL, whereas the terminals from $\mathrm{G} / \mathrm{R}$ single cones are located in the second stratum and the terminals from $\mathrm{B} / \mathrm{V}$ single cones are located in the third stratum (Fig. 1F). 

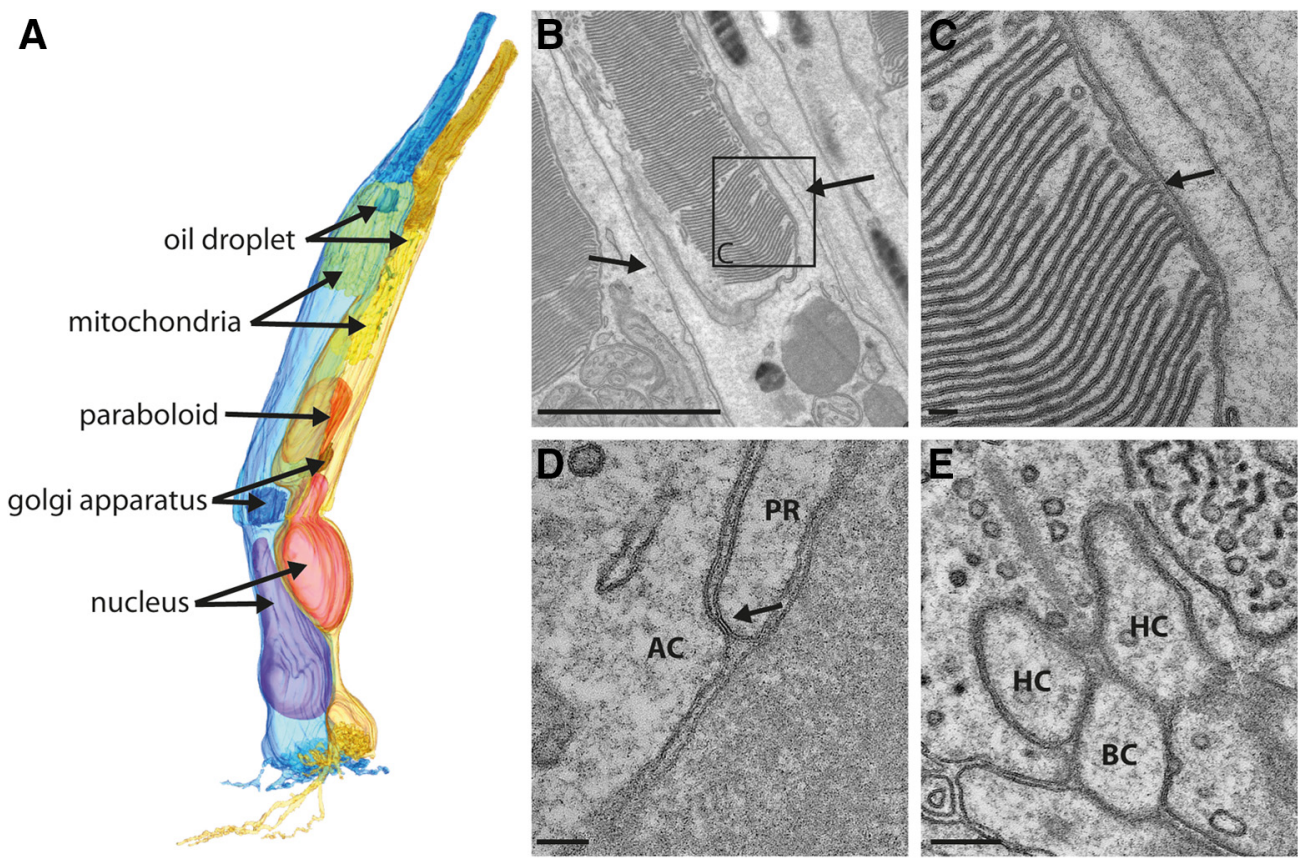

Figure 2. Double-cone anatomy in the chicken retina. $A, 3 D$ reconstruction of both members of a double cone, the principal member (blue) and the accessory member (pale orange), from the outer segments containing densely packed disk membranes to the photoreceptor terminal where the signal transfer occurs. The oil droplets in both members (green) are located in the inner segments directly at the border to the outer segments. In the accessory member, multiple small oil droplets or granules could be found. The $>200$ mitochondria/cell (yellow) are densely packed in the ellipsoid part of the inner segment. The Golgi apparatus (red) is located in the myoid part of the inner segment close to the nucleus (magenta). The accessory member of the double cone additionally contains a paraboloid (dark orange) in the myoid part of the inner segment. $\boldsymbol{B}$, Transmission electron microscopy (TEM) image of the outer segment of an accessory member from a double cone. Densely packed discs are visible as well as two of the multiple oil droplets and calyceal processes on both sides of the outer segment, as indicated by the arrows. C, Magnified area from $\boldsymbol{B}$ reveals the typical invaginations formed in the outer segments of cones (arrow). $\boldsymbol{D}$, TEM images of the outer membranes of both double-cone members, which form junction-like structures (arrow) along the inner segments. $\boldsymbol{E}$, TEM image of a ribbon synapse in the principal member of a double-cone terminal. $\mathrm{HC}$, horizontal cell dendrite; $\mathrm{BC}$, invaginating bipolar cell dendrite. Scale bars: $\boldsymbol{B}, 2 \mu \mathrm{m} ; \boldsymbol{C}, \boldsymbol{D}, 100 \mathrm{~nm} ; \boldsymbol{E}, 200 \mathrm{~nm}$.

\section{Morphologic characterization of the double cone}

With the high-resolution $3 \mathrm{D}$ reconstruction of a complete double cone, including all its major compartments, we reinvestigated the double-cone anatomy in the chicken retina (Fig. $2 \mathrm{~A}$, subvolume 1, Extended Data Fig. 1-1F). We could identify multiple tiny oil droplets in the accessory member of the double cone and a single oil droplet in the principal member, as indicated by Pedler and Boyle (1969). Because of the small size of the multiple oil droplets in the accessory member, they may have been overlooked in light microscopic studies (López-López et al., 2008), leading to different opinions on whether or not the accessory member of double cones has a fractionated oil droplet. The principal member has a mean number of $9.1 \pm 3.2$ (mean \pm SD; $n=14$ ) processes, which spread radially but never exceed the first stratum of the OPL. In contrast, the accessory member extends several short processes toward the principal member and additionally $2.8 \pm 0.7(n=14)$ long processes, which pass underneath the terminal of the principal member and can reach the third stratum of the OPL (Fig. 2A). Processes were only analyzed from double-cone members where the complete terminal was in the volume.

For an even more detailed view on specific structures in the double cone, we used transmission electron microscopy. By analyzing the highly stacked and ordered membranes in the outer segments of the double cone, we validated the tissue preservation (Fig. $2 B$ ) and were able to visualize the outer membrane invaginations (Fig. 2C). A closer look into the opposing membranes of the double-cone members confirmed junction-like structures along the inner segments of the double cones (Fig. 2D), which may represent gap junctions, as described by Nishimura et al.
(1981). Additionally, we observed that only the accessory member of the double cone expressed a paraboloid, which is a region of accumulated glycogen surrounded by a large density of endoplasmic reticuli (Cohen, 1972). However, we did not find any specialized structure that could have evolved specifically for the putative functions of chicken double cones in magnetoreception and/or polarized light vision.

\section{Classification of photoreceptor contacts}

Having confirmed the morphology of double cones in the chicken, we investigated how double cones are connected to other photoreceptors and how the photoreceptors are connected to bipolar cell types. To characterize connecting cells, we first determined what is an actual contact and which type of contact cells make with each other. However, the typical morphology of ribbon synapses (Meller, 1964; Dowling and Boycott, 1966; Lagnado and Schmitz, 2015) and basal (flat) contacts (Dowling and Boycott, 1966; Haverkamp et al., 2000) in the photoreceptor terminals could still be identified and were used to classify the type of contact (for details, see Materials and Methods). Ribbon synapses are located inside the photoreceptor terminal (Fig. 2E). Two lateral horizontal cell dendrites and one or two central bipolar cell dendrites invaginate the photoreceptor terminal at sites where ribbons are anchored (Dowling and Boycott, 1966). In general, ON bipolar cells usually make invaginating (central) contacts, and OFF bipolar cells usually make basal (flat) contacts at the base of the photoreceptor terminal (Haverkamp et al., 2000). But there are several exceptions, where OFF bipolar cells occupy the central part of a ribbon synapse (fish; Saito et al., 1985) or ON bipolar cells make basal contacts (Calkins et al., 
front view
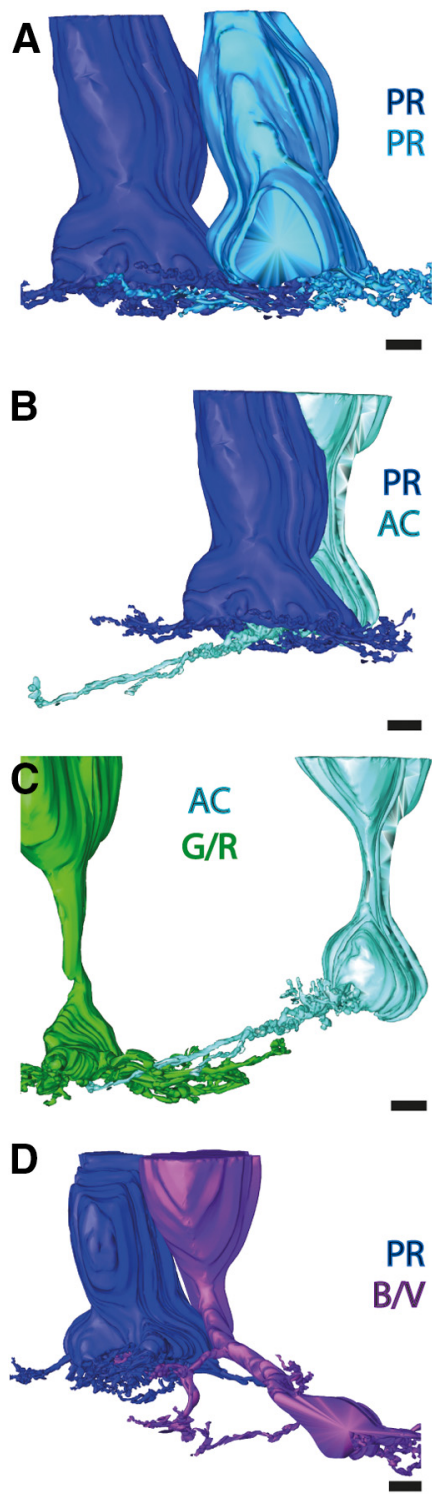

E

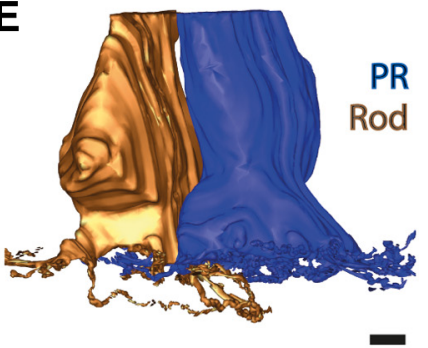

PR bottom view
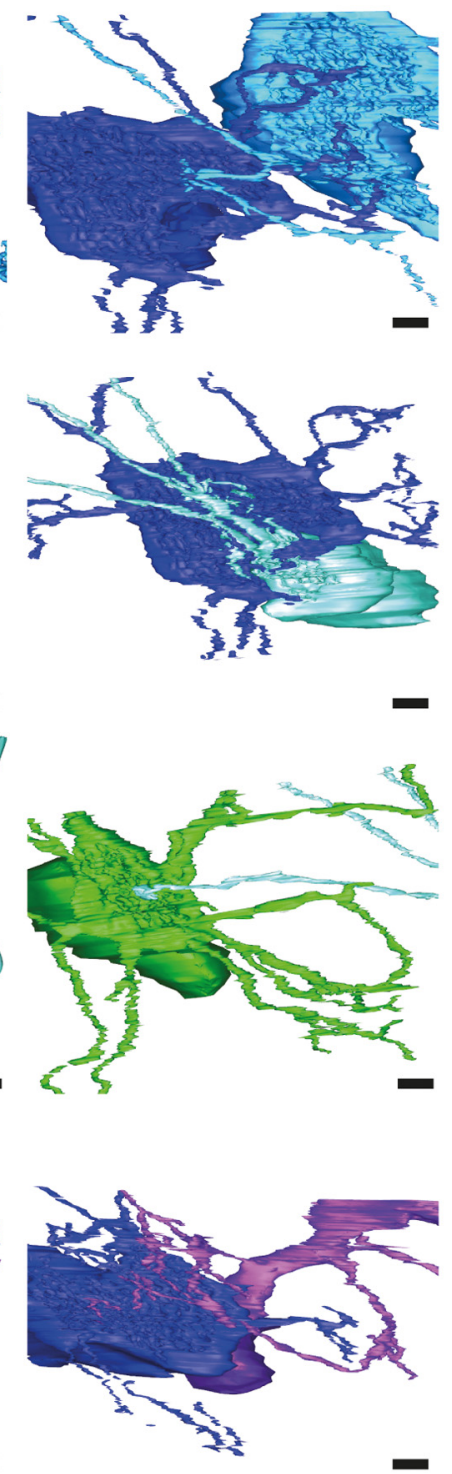

$-$

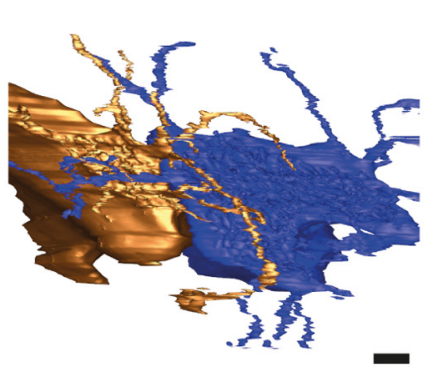

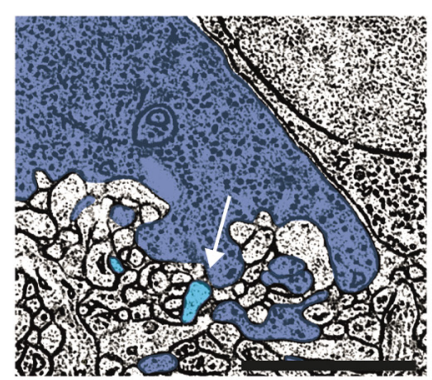
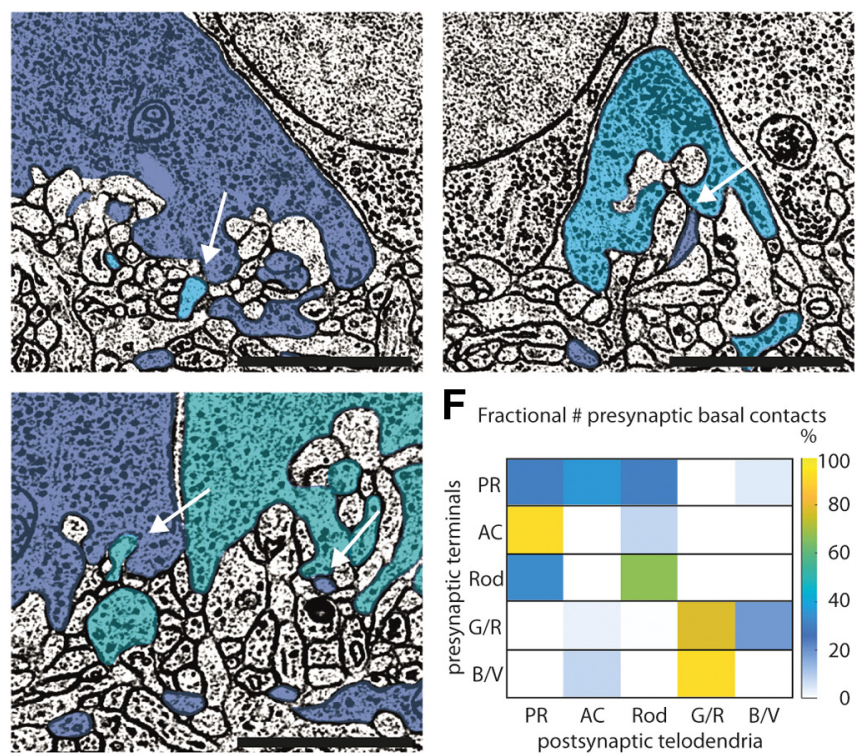

F Fractional \# presynaptic basal contacts

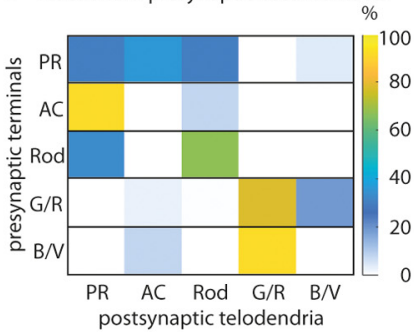

G Fractional \# postsynaptic basal contacts

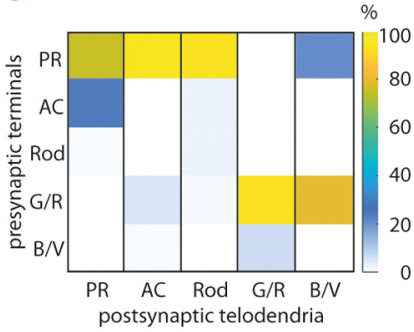

H Fractional \# presynaptic ribbon contacts

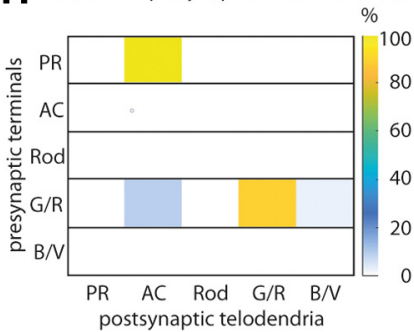

I Fractional \# postsynaptic ribbon contatts

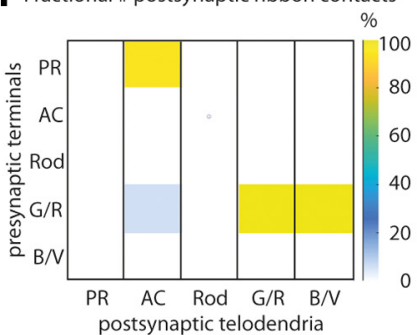

Figure 3. Connections from the double cone to other photoreceptors. The two images on the left show front and bottom views of two neighboring 3D reconstructed photoreceptor terminals. $A$, Contacts between the PRs of neighboring double cones (dark and bright blue). The electron microscopy (EM) images on the right show that both PRs make basal contacts in the neighboring PR terminal (white arrows). $B$, Contacts between the AC (turquoise) and PR (dark blue) of a double cone. The EM image on the right shows that double-cone members make basal contacts in the other member's terminal, and that the $\mathrm{AC}$ additionally makes contacts through ribbon synapses in the PR terminal (white arrows). $C$, Contact between the AC (turquoise) and a G/R cone (green). The two images on the left indicate that only the $A C$ makes a contact to the $G / R$ cone terminal but not the other way around. The EM image on the right shows that the $A C$ makes a ribbon synapse contact to the $G / R$ cone terminal. $D$, Contact between the PR of a double cone and a $B / N$ cone. The two images on the left indicate that only the $B / N$ cone makes contacts to the PR terminal, whereas the PR does not seem to contact the B/N cone terminal. The EM image on the right shows that the $B / N$ cone makes multiple basal contacts to the PR terminal. $E$, Contacts between PR and rods. The EM images show that only the rod makes a basal contact with the PR terminal, but not the other way around. $F$, Connectivity matrix with percentage representations (see Materials and Methods) of presynaptic basal contacts from PR (total contacts $=248$ ), AC (total contacts $=26$ ), rods (total contacts $=3$ ), $G / R$ (total contacts $=180$ ), and B/N (total contacts $=12) . G$, Connectivity matrix with percentage representations of postsynaptic basal contacts from PR (total contacts $=100$ ), $A C$ (total contacts $=96)$, rods (total contacts $=79$ ), $G / R$ (total contacts $=151$ ), and $B / V$ (total contacts $=43$ ). $\boldsymbol{H}$, Connectivity matrix with percentage representations of presynaptic ribbon contacts from PR (total contacts $=45$ ), $A C$ (total contacts $=0$ ), rods (total contacts $=0), G / R$ (total contacts $=35$ ), and $B / V($ total contacts $=0$ ). $I$, Connectivity matrix with percentage representations of postsynaptic ribbon contacts from PR 
1996). In the turtle, the lateral or central positions could also be occupied by a process from another photoreceptor (Mariani and Lasansky, 1984).

\section{The number of ribbon synapses}

We first counted the number of ribbon synapses expressed in a subset of rod and cone terminals. Since the terminals of green and red cones as well as blue and violet cones terminate in the second and third strata of the OPL, respectively, the number of ribbons provided below is the average number for the respective two types combined. In contrast to rodent rods, which only express one ribbon per terminal (Ladman, 1958; Cohen, 1960), rods from chickens contained $3 \pm 0.8(n=20)$ ribbons per terminal. In double cones, the mean number of ribbons was larger in the principal member $(18.5 \pm 2.0, n=20)$ than in the smaller accessory member $(8.7 \pm 0.8, n=20)$. The terminals of single cones contained a similar number of ribbons (green/red single cones: $9.5 \pm 1.4, n=20$; blue/violet single cones: $8.9 \pm 1.3$, $n=10$ ). Ribbon synapses were only counted in complete terminals of rods and cones throughout the dataset.

\section{Identification of photoreceptor/photoreceptor connections}

Telodendria are present at the photoreceptor terminals of various vertebrate species [e.g., cats (Kolb, 1977), primates (O’Brien et al., 2012), zebrafish (Li et al., 2009; Noel and Allison, 2018), and birds (Mariani and Leure-du Pree, 1978)]. While telodendria were shown to be coupled by gap junctions in a number of species [e.g., ground squirrel (DeVries et al., 2002), primate (Hornstein et al., 2005), and zebrafish (Noel and Allison, 2018)], telodendria of turtle photoreceptors invaginate into neighboring terminals, making chemical contacts at the ribbon synapse (Mariani and Lasansky, 1984; Kolb and Jones, 1985; Owen, 1985; Ammermüller and Kolb, 1996).

The analysis of photoreceptor/photoreceptor contacts was performed in subvolume $2(140 \times 148 \times 10.4 \mu \mathrm{m}$; Extended Data Fig. 1-1F). By analyzing tip-to-tip and tip-to-shaft telodendria contacts (Extended Data Fig. 3-1A,B, definition), which most likely represent gap junctions, we found that tip-to-tip contacts only occurred between cells of the same cell type, predominantly between rods and in smaller numbers also between the principal members of the double cone and green/red single cones (Extended Data Fig. 3-1C). Tip-to-shaft telodendrial contacts were detected between cells from the same type but also between cells from different photoreceptor cell types, with the highest numbers being observed between different single-cone types (Extended Data Fig. 3-1D). Although telodendria are abundant in the chicken OPL, telodendria-telodendria contacts are rather restricted (18.4\% of the total number of detected contacts), and most of the photoreceptor telodendria make contact within the terminals of other photoreceptors.

However, as in the turtle, chicken photoreceptor telodendria frequently contacted other photoreceptor terminals via basal and ribbon contacts, which looked similar to the basal and ribbon contacts between bipolar cells and photoreceptor cells described above. The accessory members of double cones are highly

(total contacts $=0$ ), AC (total contacts $=48$ ), rods (total contacts $=0$ ), G/R (total contacts = 31), and B/V (total contacts = 1). Tip-to-tip and tip-to-shaft photoreceptor contacts are shown in Extended Data Figure 3-1, and the synaptic connectivity of a principal member terminal is shown in Extended Data Figure 3-2. All photoreceptor cell contacts are shown in Extended Data Figures 3-3, 3-4, 3-5, 3-6. Scale bars, $2 \mu \mathrm{m}$. connected to their affiliated principal member by telodendrial basal and ribbon contacts in the terminal of the principal member (Fig. 3B, $F-I$ ). In contrast, the principal member is less connected, making only basal contacts with its affiliated accessory member and no ribbon contacts at all (Fig. $3 B$, right, $F-I$ ). Principal members also made basal contacts to the terminal of other principal members (Fig. $3 A, F-I$ ). In contrast, accessory members from different double cones never made any contacts with each other (Fig. $3 F-I$ ).

Because of the length of the descending telodendria of the accessory members, we could not identify all contacts because the processes reached the end of the volume. Only in six AC cells did we identify either basal or ribbon contacts directly to green/ red cone terminals (Fig. 3C, $F-I$ ). In one case, we found a long telodendrion of an accessory member making a basal contact to a blue/violet cone (Fig. 3F,G).

Reconstructing the group of blue/violet cones, we discovered that these single cones not only have long telodendria at their terminals but also extend shorter telodendria into the first stratum of the OPL. However, some primary telodendria reached the end of the volume, and therefore the area in the first stratum could not be completely reconstructed. Nonetheless in three of the cells, we found that these shorter telodendria made basal contacts at the terminal of the principal member of double cones (Fig. $3 D, F, G)$. We found that $\mathrm{B} / \mathrm{V}$ single cones do not contact each other but rather contact G/R single cones (Fig. $3 F-I$ ).

Almost all rods (90\%) contacted the terminal of principal members of double cones with their telodendria via basal contacts (Fig. 3E,F,G). Although these rods received major input from the principal member, it is interesting to note that these rods were mostly presynaptic to other rods, representing an asymmetry in signal transmission.

However, immunohistological staining of connexins, the building blocks of gap junctions, indicated that gap junctions could also be present within the terminals of photoreceptors in the chicken retina (Kihara et al., 2009). Therefore, it could be possible that telodendria and photoreceptor terminals form not only chemical synapses, but also gap junctions.

In summary, these analyses reveal a surprisingly complex network of connections among bird photoreceptors, suggesting that the bird retina is more similar to other sauropsida retinas such as the turtle (Mariani and Lasansky, 1984) than to the mammalian (Hornstein et al., 2005) or fish retina (Noel and Allison, 2018) in this regard. All individual photoreceptor contacts are listed in Extended Data Figs. 3-3, 3-4, 3-5, 3-6.

\section{Identification of bipolar cell types}

Since the connectivity between photoreceptors and bipolar cells is unknown for birds and because the number of previously anatomically described bipolar cell types in the bird retina seemed surprisingly low (11 in chicken, 8 in pigeon; Mariani, 1987; Quesada et al., 1988), in subvolume 2 (Extended Data Fig. 1-1F) we reconstructed all bipolar cells that contacted at least one of the reconstructed photoreceptors. Bipolar cells that had their main dendritic and axonal fields inside the volume were considered as complete cells $(n=74)$. These cells were used for identifying the connectivity between bipolar cells and photoreceptor cells and for defining bipolar cell types. Bipolar cells, of which the primary axon reached the end of the volume or major parts of the dendritic field were missing, were defined as partial bipolar cells $(n=72)$ and were only further analyzed if they could be clearly assigned to one bipolar cell type (e.g., based on contacted photoreceptor types, fitting into the existing 


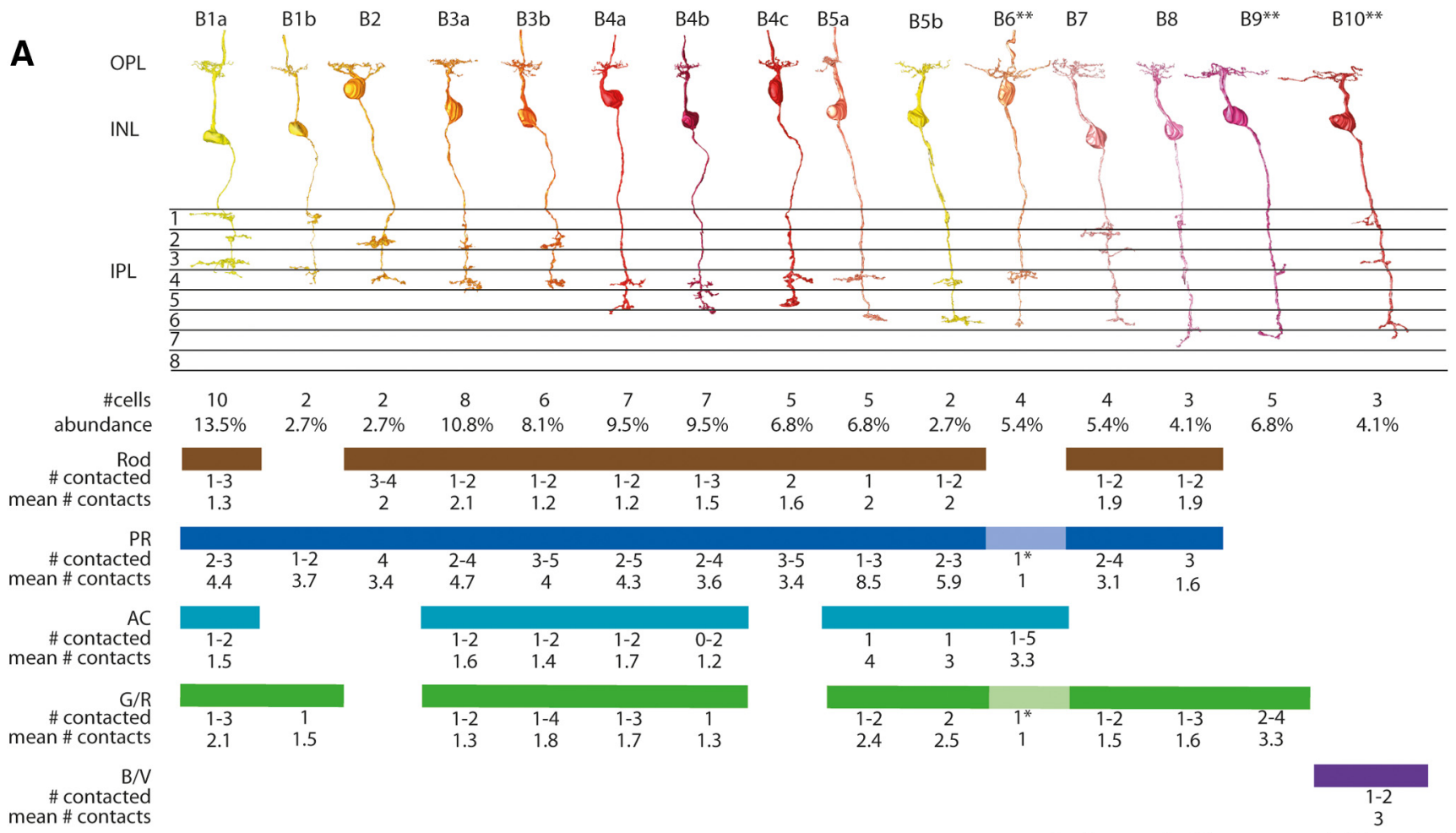

\section{B}

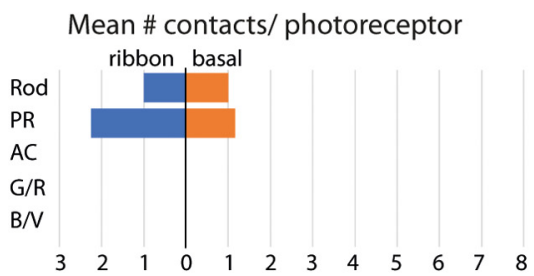

\section{C}

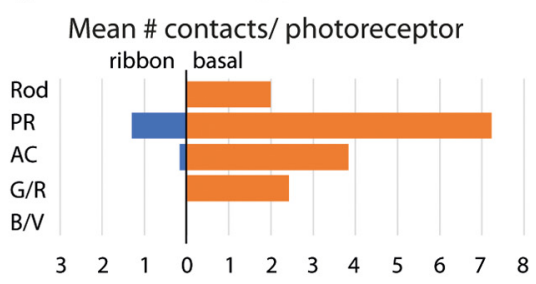

$\mathbf{F}$

$\mathrm{B} 5 \mathrm{a}$
E

B2

Percentages of three classes of basal contacts Percentages of three classes of basal contacts

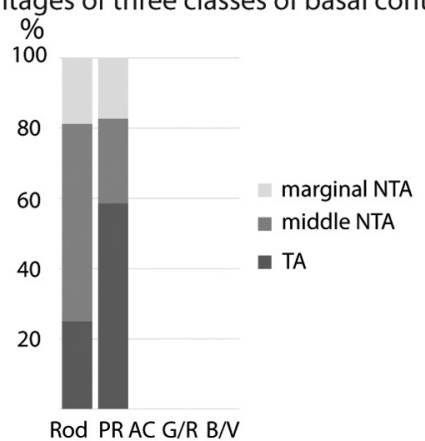

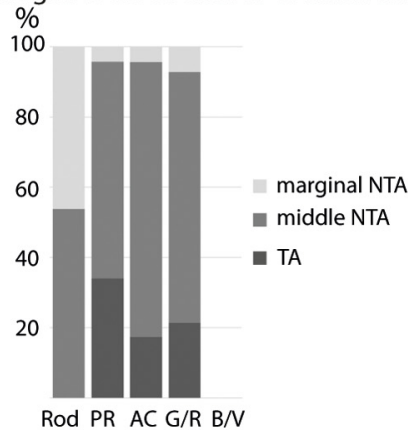

* only one cell of the group made this contact ** long unbranched dendrites, a lot reached end of volume

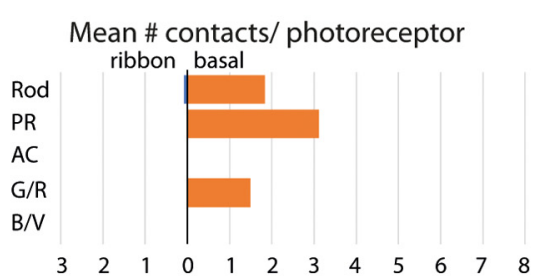

G

B7
Percentages of three classes of basal contacts

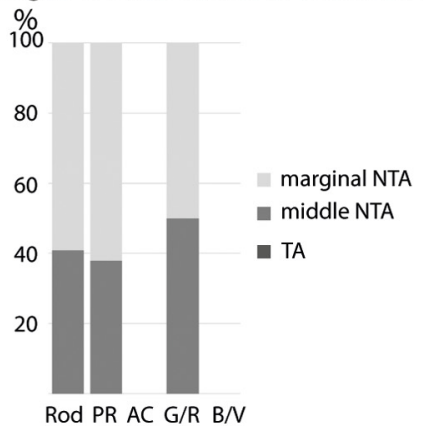

Figure 4. Bipolar cell types in the chicken retina. $\boldsymbol{A}$, Bipolar cell types are sorted based on their axonal stratification level in the IPL. Below each bipolar cell type, the numbers of cells within this cell type are shown, including their abundance within the bipolar cell class. Contacted photoreceptor types are highlighted with respective color. Number of contacted photoreceptor cells and the combined mean number of basal and ribbon contacts per photoreceptor below. $\boldsymbol{B}-\boldsymbol{D}$, Mean numbers of ribbon and basal contacts per photoreceptor terminal for bipolar cell types B2, B5a, and B7. Data are also shown in Extended Data Figures 4-3, 4-9, and 4-12 for overall bipolar cell type summary. $\boldsymbol{E}-\mathbf{G}$, Histograms showing the averaged proportion of the three different classes of basal contacts (triad-associated, TA; marginal and middle non-triad associated, NTA) of bipolar cell types B2, B5a, and B7. Data also shown in Extended Data Figures 4-3, 4-9, and 412 in the overall bipolar cell type summary. Analyses of each bipolar cell type are represented in Extended Data Figures 4-1, 4-2, 4-3, 4-4, 4-5, 4-6, 4-7, 4-8, 4-9, 4-10, 4-11, 4-12, 4-13, 4-14, 4-15, 4-16, and a comparison between identified bipolar cells in this study and other publications is shown in Extended Data Figure 4-17.

dendritic mosaic). Dendrites that contacted photoreceptors but were not connected to a soma were ignored. To identify the stratification level of the axons in the inner plexiform layer (IPL), we chose to divide the IPL into eight strata based on an earlier study analyzing the stratification of ganglion cells in chicken retina (Naito and Chen, 2004).

With the dataset at hand, we not only analyzed the morphology (dendritic and axonal stratification, soma position) of the 

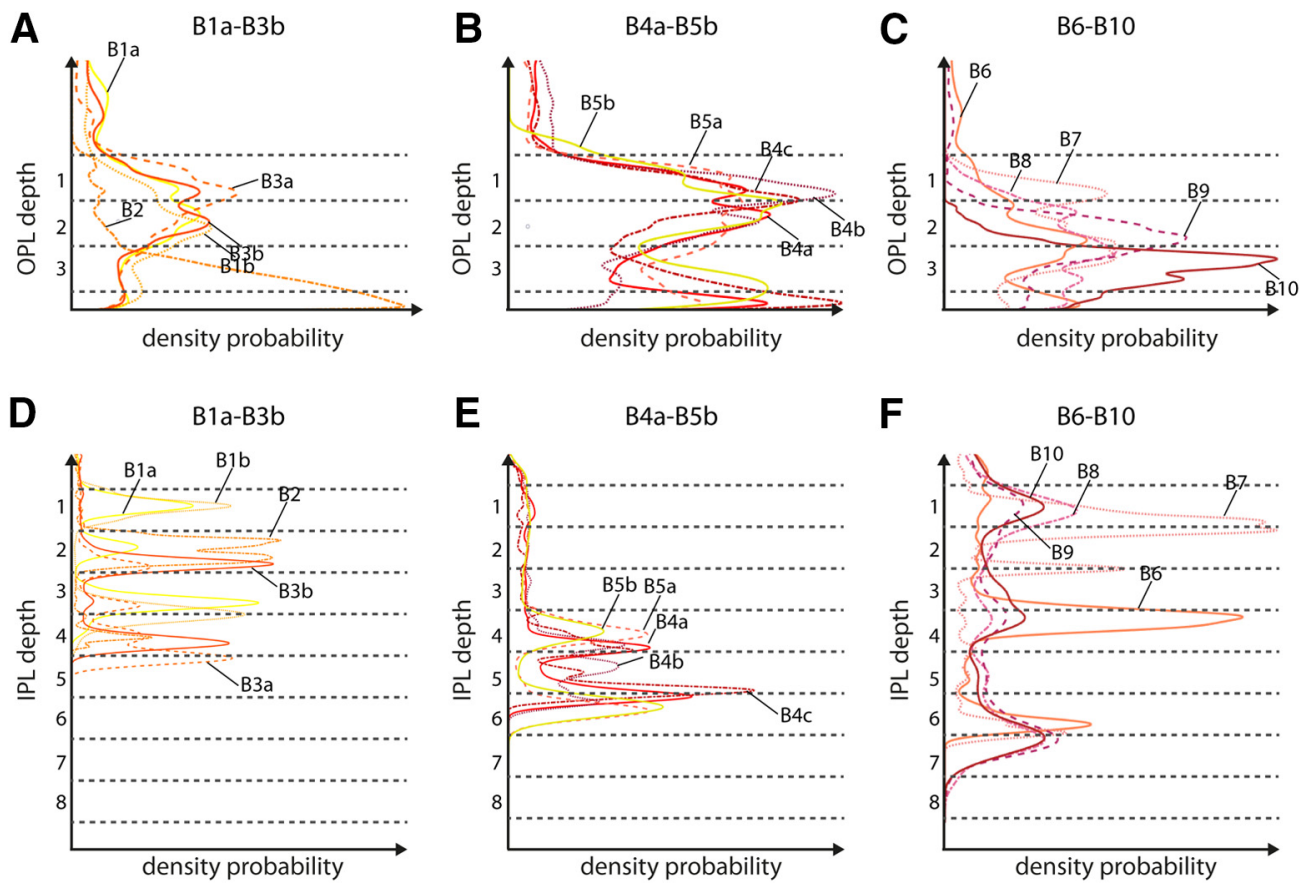

Figure 5. Stratification level of the individual bipolar cell types in the OPL and IPL. A-C, Bipolar cell stratification profile in the OPL represented by a probability density function estimate for bipolar cell types B1a-B3b, B4a-B5b, and B6-B10, respectively. Density functions are calculated based on the volume of the cells along OPL depth. Dendrites increase in volume with increasing distance to the connected terminals. Therefore, the OPL density estimate is slightly shifted toward the INL, and the peak density estimate does not represent the area of synaptic contacts. $\mathbf{D}-\boldsymbol{F}$, Bipolar cell stratification profile in the IPL represented by a probability density function estimate for bipolar cell types B1a-B3b, B4a-B5b, and B6-B10, respectively.

bipolar cells, but also their contacts to the different photoreceptor types to define bipolar cell types. In a final step, we double checked the different types by analyzing the dendritic and axonal field mosaics as described for bipolar cells from other species, such as mouse (Wässle et al., 2009; Tsukamoto and Omi, 2017) and primates (Boycott and Wässle, 1991). In total, we identified 15 types of bipolar cells in the chicken retina, sorted by the position of their axonal stratification (Fig. 4A). Detailed information on each bipolar cell type is given in Extended Data Figs. 4-1, 4-2, 4-3, 4-4, 4-5, 4-6, 4-7, 4-8, 4-9, 4-10, 4-11, 4-12, 4-13, 4-14, 4-15. In addition, we classified two fully reconstructed bipolar cells as "orphan" because they could not be assigned to any other bipolar cell type and are also different from each other (Extended Data Fig. 4-16).

For all bipolar cells, we analyzed the type of contact they made to photoreceptor terminals (ribbon vs basal contacts). We further categorized the basal contacts based on their distance to the nearest ribbon synapse (Extended Data Figs. 4-1F, 4-2F, $4-3 F, 4-4 F, 4-5 F, 4-6 F, 4-7 F, 4-8 F, 4-9 F, 4-10 F, 4-11 F, 4-12 F$, $4-13 F, 4-14 F, 4-15 F)$. Examples of this analysis are shown in Figure $4 B-G$ with B2 as a potential ON bipolar cell type, B5a as a potential midget bipolar cell type, and B7 as a potential OFF bipolar cell type. We also analyzed whether bipolar cells contacted all the photoreceptors of a specific type within their dendritic field and found that no bipolar cell type seemed to avoid a terminal of a photoreceptor type it normally contacts (Extended Data Figs. 4-1G, 4-2G, 4-3G, 4-4G, 4-5G, 4-6G, 4-7G, 4-8G, 4-9G, 4-10G, 4-11G, 4-12G, 4-13G, 4-14G, 4-15G). We calculated the stratification density probabilities of each individual bipolar cell type based on the calculated volume of the cells along retina depth (Fig. 5) reflecting the overall stratification pattern in the OPL and IPL. To visualize how much volume each bipolar cell contributes to the different strata in the IPL, we calculated the mean volume of all cells from a bipolar cell type along IPL depth
(Extended Data Figs. 4-1B, 4-2B, 4-3B, 4-4B, 4-5B, 4-6B, 4-7B, 4$8 B, 4-9 B, 4-10 B, 4-11 B, 4-12 B, 4-13 B, 4-14 B, 4-15 B$, colored lines) and calculated the sum of all bipolar cells (Extended Data Figs. 4- $1 B, 4-2 B, 4-3 B, 4-4 B, 4-5 B, 4-6 B, 4-7 B, 4-8 B, 4-9 B, 4-10 B$, $4-11 B, 4-12 B, 4-13 B, 4-14 B, 4-15 B$, black line), which enabled us to identify the overall distribution of bipolar cells along IPL depth. We found that strata 3, 5, and 8 have the lowest density of bipolar cell terminals. However, we cannot exclude that we are missing bipolar cell types because our dataset is limited in the $z$ direction.

In the OPL, we found some exclusive connections between bipolar cells and photoreceptors. Surprisingly, only one type (B9) exclusively contacts green/red single cones and one type (B10) exclusively contacts blue/violet cones. B10 bipolar cells may represent the "blue bipolar cell" known from mammalian species [e.g., mouse (Haverkamp et al., 2005), primate (Mariani, 1984; Ghosh et al., 1997), and ground squirrel (Li and DeVries, 2006)], but we would have had to be able to separate blue from violet single cones in the dataset to clearly identify single cone-type specific bipolar cells. One type (B6) seems to contact almost exclusively the accessory member of double cones (Fig. $4 \mathrm{~A}$, Extended Data Fig. 4-11). All other bipolar cell types (except for B9 and B10) make contacts with several photoreceptor cell types but receive most of their input from the principal member of double cones (Extended Data Figs. 4-1, 4-2, 4-3, 4-4, 4-5, 4-6, 47, 4-8, 4-9, 4-10, 4-11, 4-12, 4-13). Interestingly, rods make most of their ribbon synaptic contacts to B2 bipolar cells (Extended Data Fig. 4-3). Additionally, 12 of the 15 identified bipolar cells receive input from rods.

\section{Identification of the ON-OFF border in the IPL}

We were able to identify 15 different bipolar cell types, including their connectivity to photoreceptor cells. Since electrophysiological data from avian bipolar cells are completely lacking, we aimed 

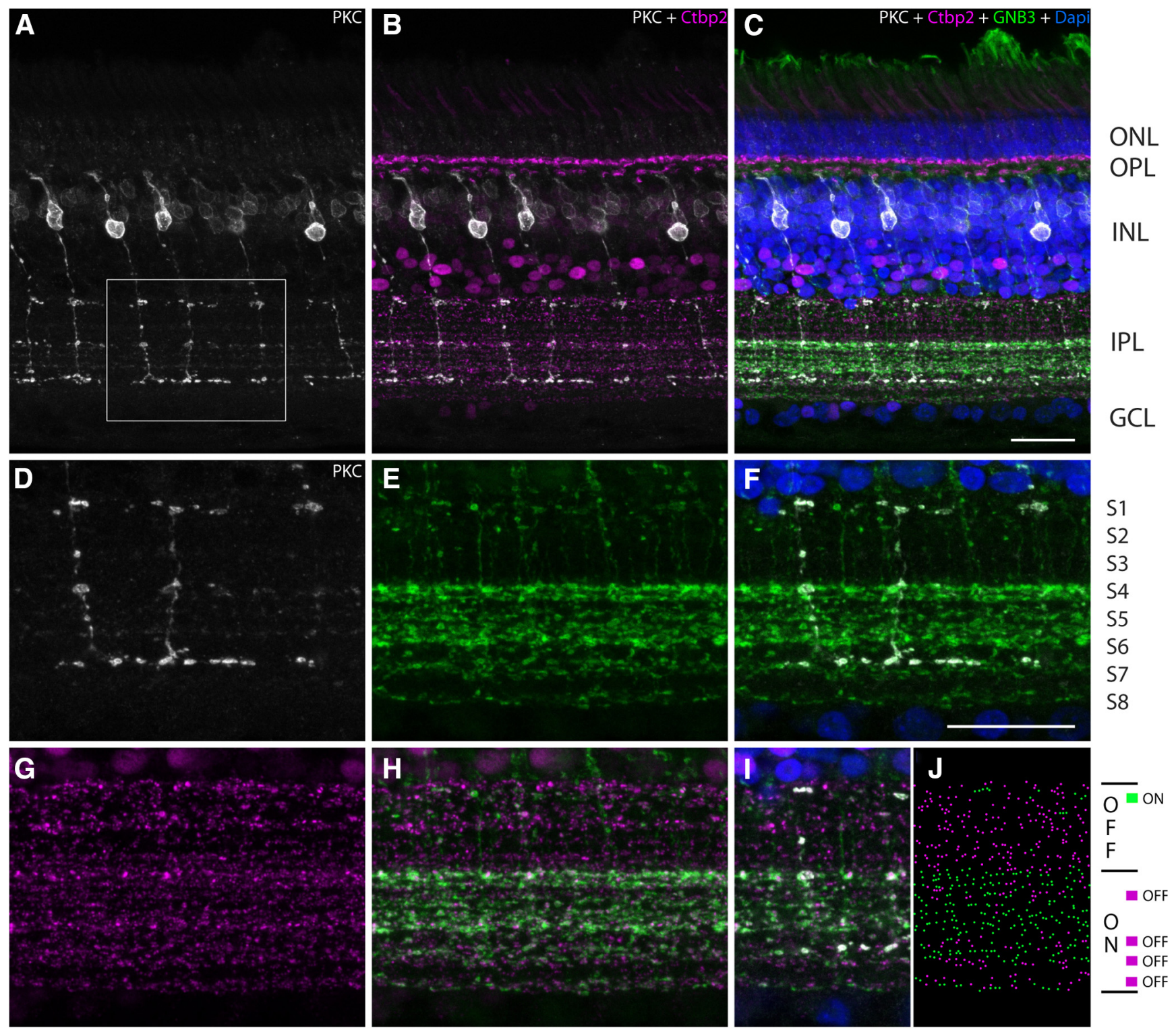

Figure 6. IPL stratification in the chicken retina. $\boldsymbol{A}-\mathbf{I}$, Vertical vibratome sections of the chicken retina were labeled with PKC (gray; $A-D, F, I)$, Ctbp2 (magenta; $\boldsymbol{B}, \boldsymbol{C}, \mathbf{G}-\boldsymbol{I})$, GNB3 (green; $\boldsymbol{C}$, $\boldsymbol{E}, \boldsymbol{F}, \boldsymbol{H}-\boldsymbol{I}$ ), and DAPI (blue; $\boldsymbol{C}, \boldsymbol{F}, \boldsymbol{I})$. PKC-labeled bipolar cell axon terminals are also GNB3 positive in the IPL indicating that these bipolar cells (high similarity with B9 and B10 in Fig. 4) are 0N bipolar cells $(\boldsymbol{D}-\boldsymbol{F})$. G-J, Double labeling with Ctbp2 and GNB3 reveals ON bipolar axon terminals in the presumed OFF layer and OFF axon terminals in the presumed ON layer of the IPL. Complete stack is available in Extended Data Figure 6-1. Scale bars: $\boldsymbol{C}$ (for $\boldsymbol{A}-\boldsymbol{C}), \boldsymbol{F}$ (for $\boldsymbol{D}-\boldsymbol{J}), 20 \mu \mathrm{m}$.

to identify a putative border between the OFF and ON strata in the IPL using immunohistochemistry to analyze which of the identified cell types may represent ON or OFF bipolar cells. Although this border is well described for mammalian species, it remains unclear in birds since even the number of strata is not entirely resolved (Millar et al., 1987; Naito and Chen, 2004; Ritchey et al., 2010). We used antibodies against PKC $\alpha$ to label a subpopulation of putative ON bipolar cells (Ritchey et al., 2010; Fig. 6, gray), GNB3 as a putative marker for all ON bipolar cells (Ritchey et al., 2010; Fig. 6, green), and Ctbp2 to visualize all ribbon synapses in our tissue (Fig. 6, magenta). PKC-positive cells were found to be positive for GNB3, indicating that these cells are indeed $\mathrm{ON}$ bipolar cells (Fig. 6A-F). Comparing the morphology and stratification levels of PKC-positive bipolar cells with our 3D reconstructed cells, we found a high similarity with B9 and B10 bipolar cells, which exclusively contact single cones. The only difference between the PKC-positive cells and the B9 or B10 bipolar cell types, respectively, is the stratification in the innermost stratum (S6/7), which is more excessive in the PKC cells than in our reconstructions. Additionally, if the GNB3 marker indeed labels the ON strata of the avian IPL and we assume eight strata, then the border between OFF and ON would be between S3 and S4 (Fig. 6E,F). Comparing the GNB3 staining with Ctbp2 labeling, we found that synapses in the supposed OFF stratum 1 are GNB3 positive, which indicates that ON bipolar cells also stratify in the distal OFF layer. Likewise, we found Ctbp2-positive but GNB3-negative areas between S4 and S5, and S6 and S7, and in S7 and S8, indicating that OFF bipolar cells stratify in the ON strata of the IPL as well (Fig. 6I,J). This eliminates the clear separation between $\mathrm{ON}$ and OFF signals in the IPL. If we apply this stratification pattern to our reconstructed bipolar cells and combine it with the type of contacts a bipolar cell makes to the photoreceptor terminals, we suggest that bipolar cells belonging to our groups B1, B3, B7, and B8 are putative OFF bipolar cells, whereas the groups of B2 to B6, B9, and $\mathrm{B} 10$ are putative $\mathrm{ON}$ bipolar cell candidates. Clearly, 
electrophysiological studies are needed to corroborate these putative assignments.

\section{Discussion}

We obtained the first 3D electron microscopic dataset for the avian retina using ssmSEM. We used this dataset to (1) revisit the anatomy of double cones, (2) present insights into doublecone connectivity to other photoreceptors, and (3) provide the first classification of avian bipolar cell types that considers photoreceptor-bipolar cell connectivity. Additionally, we found a surprisingly large and diverse number of photoreceptor-photoreceptor connections, which, to our knowledge, were not previously described in such diversity in any bird, and a surprising number of inputs from several different photoreceptor cells to most of the individual bipolar cell types.

\section{Photoreceptor-photoreceptor connections}

Evidence from mammals and fish suggests that telodendria contact each other and form gap junctions (DeVries et al., 2002; Noel and Allison, 2018), which are thought to have various functions. Cone-cone coupling, for example, correlates common light-mediated input and reduces noise from individual photoreceptors by averaging across the coupled network (DeVries et al., 2002; O'Brien et al., 2012), thereby increasing the signal-to-noise ratio. Rod-rod coupling was shown to enhance contrast detection in dim light, at the cost of reducing absolute sensitivity (Li et al., 2012). Here, we found that rod photoreceptors predominantly form tip-to-tip and tip-to-shaft telodendrial contacts (Extended Data Fig. 3-1). Since we were not able to identify gap junctions in our ssmSEM dataset, we can only speculate that rod-rod electrical coupling is more abundant than cone-cone or rod-cone coupling in the chicken and that they may serve similar functions as in other vertebrate classes.

In turtles, rods and cones also form chemical synapses between telodendria and photoreceptor terminals (Mariani and Lasansky, 1984; Kolb and Jones, 1985; Owen, 1985). Functional experiments in tiger salamander also suggest that rods and cones are electrically and chemically coupled (Attwell et al., 1983). Here, we report for the first time in birds, that chicken photoreceptor cells also make telodendrial contacts with other photoreceptor terminals. These contacts mainly involved one or both members of a double cone and other photoreceptor cell types, but also occurred between green/red single cones.

Our data suggest that rod-rod coupling in the chicken retina could primarily be mediated by electrical synapses, whereas cone-cone or rod-cone connections could be mediated by chemical synapses. In turtles, sites of chemical synapses between red and green cones were hypothesized to lead to an excitatory color mixing, which was found in electrophysiological recordings (Kolb and Jones, 1985). Since rods and double cones are likely to be involved in luminance detection rather than color vision (Lind et al., 2014), the chemical synapses between these two photoreceptor types could serve to enlarge the operational range of the luminance channel and thereby enhance its sensitivity.

\section{Bipolar cell classification and connectivity to photoreceptor cells}

The bipolar cell classification provided here comprises 15 different types. Interestingly, we found that the axonal stratification pattern and photoreceptor connectivity were the most defining factors for the types: bipolar cells with the same axonal arbor contacted different photoreceptors. This is similar to the zebrafish retina (Li et al., 2012), but in contrast to the mammalian retina where the same axonal stratification is associated with the same photoreceptor connectivity (Euler et al., 2014). The presence or absence of a Landolt's club does not seem to be a defining feature because cells belonging to the same bipolar cell type, based on uniform dendritic and axonal mosaics, can possess, or lack this special protrusion (e.g., types 3a, 4c; Extended Data Figs. 4-4, 4-5, 4-6, 4-7,4-8). If the Landolt's club was a defining feature, we would expect the mosaics to show higher inhomogeneity and overlap within our defined bipolar cell types.

For the retina of a tetrachromatic animal (Hart, 2001), it seems very surprising that most bipolar cells make contacts to different photoreceptor cell types, thereby mixing color channels. Furthermore, most bipolar cell types seem to get most of their input from the principal member of double cones (Fig. $4 A$, Extended Data Figs. 4-1, 4-2, 4-3, 4-4, 4-5, 4-6, 4-7, 4-8, 4-9, 4-10, 4-11, 4-12, 4-13, 4-14, 4-15). This may indicate that double cones are involved not only in luminance detection, but also in color vision, presumably processed in parallel bipolar cell pathways. Bipolar cells in other animals contacting exclusively one type of single cone usually have wide dendritic fields [e.g., in rabbit (MacNeil and Gaul, 2008) and mouse (Haverkamp et al., 2005)], and these are the ones in our dataset that have reached the end of the volume more frequently than small-field bipolar cells. Therefore, bipolar cells exclusively contacting individual photoreceptor types (i.e., presumed color-sensitive bipolar cells) may be underrepresented in our analysis, which could explain the low number of bipolar cell types contacting the $\mathrm{B} / \mathrm{V}$ single cones. Nevertheless, it is surprising that only one bipolar cell type contacted the $\mathrm{B} / \mathrm{V}$ single cones, whereas at least in zebrafish the UV single cones were found to be frequently contacted by a large number of bipolar cells (Li et al., 2012). Bipolar cell type B5a may represent a bird midget bipolar cell type. Primate midget bipolar cells are characterized by a very small dendritic and axonal field, and they contact only one single cone in the central retina (Polyak, 1941; Puller et al., 2007). Therefore, midget bipolar cells mediate high-acuity vision (Euler et al., 2014). Since our dataset is from peripheral chicken retina, it might be possible that this cell type receives single-cone input in the area centralis. For other vertebrate retinas, it was proposed that bipolar cell types follow a so-called "block wiring," meaning that they contact spectrally neighboring photoreceptor cells (red and green) rather than leaving a spectral gap (red and blue; for review, see Baden and Osorio, 2019). Our dataset also supports this hypothesis for the chicken retina, although we cannot separate all single-cone types.

Recently, a detailed analysis of chicken retinal cell types was performed using single-cell transcriptomics, and 22 different bipolar cells were identified (Yamagata et al., 2021), indicating that we are missing 7 bipolar cell types. One reason could be the limited $z$-depth of our dataset, which potentially leads to a reduced number of wide-field bipolar cells. In addition, separating the $\mathrm{R} / \mathrm{G}$ and $\mathrm{B} / \mathrm{V}$ groups of single cones could also lead to a regrouping of the bipolar cell types. Compared with previous anatomic studies, we were able to link all of the bipolar cell types identified in pigeons (Mariani, 1987) to our data. We also found similarities to the identified bipolar cells in chicken retina, but also identified new types (Quesada et al., 1988; Extended Data Fig. 4-17, detailed comparison).

In general, it is striking that all bipolar cell types are at least bistratified in the IPL, whereas mammalian bipolar cells are mostly monostratified (Euler et al., 2014) and fish have a few monostratified cells in addition to several multistratified types 
(Li et al., 2012). Since the border between the ON and OFF layer in the IPL is not resolved in the chicken, we used the GNB3 staining as a reference for ON bipolar cells (Ritchey et al., 2010) and Ctbp 2 as a ribbon synaptic marker. Combining the axonal stratification of the identified bipolar cells with the immunostaining and the type of contacts the cells made to the photoreceptor terminals, it is likely that OFF bipolar cells also stratify in the ON strata of the IPL as it was demonstrated in turtle retina (Ammermüller et al., 1995) and zebrafish retina (Connaughton, 2001). Our Ctbp 2 staining supports that hypothesis because we found ribbon synapses in GNB3-negative strata in the ON part of the IPL. Additionally, OFF bipolar cells can form ribbon synapses with photoreceptors (e.g., in fish; Sakai and Naka, 1983; Saito et al., 1985), and we cannot conclude that all bipolar cells that make basal contacts are OFF cells because, in primates, $\mathrm{ON}$ bipolar cells also form basal contacts with the photoreceptor terminal (Hopkins and Boycott, 1996). Thus, functional data are needed to unequivocally decide which bipolar cell types are $\mathrm{ON}$ and OFF cells.

\section{Could the region in the retina change the proportion of bipolar cell types or shapes?}

Our dataset was recorded in the dorsal area of the left eye of a 1-week-old chicken (G. gallus domesticus). The photoreceptor density and composition in the retinas of birds and other animals change from the periphery to the center and can, in some cases, be divided into special areas (for review, see Seifert et al., 2020). Not only does the ratio between the photoreceptor types change between periphery and center, but the total number of photoreceptor cells and ganglion cells also decrease from the center to the periphery (Bueno et al., 2011). The increasing number of photoreceptor cells could lead to a higher density of bipolar cells with smaller dendritic fields in the central retina. Also, we cannot exclude that the central retina harbors bipolar cell types that have been missing from the more peripheral area analyzed here. Additionally, it was reported that the left eye is more involved in high spatial acuity tasks than the right eye (for review, see Seifert et al., 2020), indicating that the retinal wiring may differ between both eyes.

\section{Double-cone anatomy}

The detailed reconstruction of an individual double cone largely confirmed previous data, as follows: (1) accessory members show fragmented oil droplets, and (2) principal and accessory members are connected by junction-like structures. These structures may, at first glance, contradict the idea that light-dependent magnetoreception could be based on the differential signaling of avian double cones by expressing two sensors arranged in different orientations (Hore and Mouritsen, 2016; Worster et al., 2017). Electrically mixing the resulting signals already at the photoreceptor level seems counterintuitive. However, as we do not know under which light conditions (Yang and $\mathrm{Wu}, 1989$ ) or times of day (Ribelayga et al., 2008) the gap junction-like structures can pass signals, these gap junctions might be closed under the light conditions when magnetoreception is most relevant. Further research should investigate this issue.

\section{Conclusion}

Here, we present the first 3D reconstructed bird retina dataset. We identified many previously undescribed photoreceptor-photoreceptor connections in the chicken retina, which suggests numerous interactions between rods and cones as well as between different cone types at the first stages of visual processing. We increased the number of morphologically known bipolar cell types from 11 to 17 and identified their complete connectivity to photoreceptor cells. Surprisingly, most bipolar cell types contact several different photoreceptor cell types. Astoundingly, 13 of 15 identified bipolar cell types got at least part of their input from double cones. Therefore, our data suggest that the double cones play many different and important roles in avian retinal processing and that the neural network and thus information processing in the outer retina are in general much more complex than previously expected.

\section{References}

Ammermüller J, Kolb H (1996) Functional architecture of the turtle retina. Prog Retin Eye Res 15:393-433.

Ammermüller J, Muller JF, Kolb H (1995) The organization of the turtle inner retina. I. ON- and OFF-center pathways. J Comp Neurol 358:1-34.

Attwell D, Werblin FS, Wilson M, Wu SM (1983) A sign-reversing pathway from rods to double cone and single cones in the retina of the tiger salamander. J Physiol 336:313-333.

Baden T, Osorio D (2019) The retinal basis of vertebrate color vision. Annu Rev Vis Sci 5:177-200.

Bowmaker JK (2008) Evolution of vertebrate visual pigments. Vision Res 48:2022-2041.

Boycott BB, Wässle H (1991) Morphological classification of bipolar cells of the primate retina. Eur J Neurosci 3:1069-1088.

Bueno JM, Giakoumaki A, Gualda EJ, Schaeffel F, Artal P (2011) Analysis of the chicken retina with an adaptive optics multiphoton microscope. Biomed Opt Express 2:1637-1648.

Calkins DJ, Tsukamoto Y, Sterling P (1996) Foveal cones form basal as well as invaginating junctions with diffuse ON bipolar cells. Vision Res 36:3373-3381.

Chernetsov N, Pakhomov A, Kobylkov D, Kishkinev D, Holland RA, Mouritsen H (2017) Migratory Eurasian reed warblers can use magnetic declination to solve the longitude problem. Curr Biol 27:2647-2651.

Cohen AI (1960) The ultrastructure of the rods of the mouse retina. Am J Anat 107:23-48.

Cohen AI (1972) Rods and cones. In: Handbook of sensory physiology VVII/ 2. Physiology of photoreceptors organs (Fuortes M, ed), pp 63-110. Berlin: Springer.

Connaughton VP (2001) Organization of ON- and OFF-pathways in the zebrafish retina: neurotransmitter localization, electrophysiological responses of bipolar cells, and patterns of axon terminal stratification. Prog Brain Res 131:161-176.

DeVries SH, Qi X, Smith RG, Makous W, Sterling P (2002) Electrical coupling between mammalian cones. Curr Biol 12:1900-1907.

Dowling JE, Boycott BB (1966) Organization of the primate retina: electron microscopy. Proc R Soc Lond B Biol Scie 166:80-111.

Drenhaus U, Morino P, Veh RW (2003) On the development of the stratification of the inner plexiform layer in the chick retina. J Comp Neurol 460:1-12.

Drenhaus U, Voigt T, Rager G (2007) Onset of synaptogenesis in the plexiform layers of the chick retina: a transmission electron microscopic study. Microsc Res Tech 70:329-335.

Eberle AL, Zeidler D (2018) Multi-beam scanning electron microscopy for high-throughput imaging in connectomics research. Front Neuroanat 12:112-117.

Eberle AL, Mikula S, Schalek R, Lichtman JW, Knothe Tate ML, Zeidler D (2015) High-resolution, high-throughput imaging with a multibeam scanning electron microscope. J Microsc 259:114-120.

Ebrey T, Koutalos Y (2001) Vertebrate photoreceptors. Prog Retin Eye Res 20:49-94.

Euler T, Haverkamp S, Schubert T, Baden T (2014) Retinal bipolar cells: elementary building blocks of vision. Nat Rev Neurosci 15:507-519.

Ghosh KK, Martin PR, Grünert U (1997) Morphological analysis of the blue cone pathway in the retina of a New World monkey, the marmoset Callithrix jacchus. J Comp Neurol 379:211-225.

Günther A, Einwich A, Sjulstok E, Feederle R, Bolte P, Koch K-W, Solov'yov IA, Mouritsen H (2018) Double-cone localization and seasonal 
expression pattern suggest a role in magnetoreception for European robin cryptochrome 4. Curr Biol 28:211-213.e4.

Hart NS (2001) The visual ecology of avian photoreceptors. Prog Retin Eye Res 20:675-703.

Haverkamp S, Grünert U, Wässle H (2000) The cone pedicle, a complex synapse in the retina. Neuron 27:85-95.

Haverkamp S, Wässle H, Duebel J, Kuner T, Augustine GJ, Feng G, Euler T (2005) The primordial, blue-cone color system of the mouse retina. J Neurosci 25:5438-5445.

Hering H, Kröger S (1996) Formation of synaptic specializations in the inner plexiform layer of the developing chick retina. J Comp Neurol 375:393405.

Higham DJ, Higham NJ (2016) MATLAB guide. Philadelphia: Society for Industrial and Applied Mathematics.

Hopkins JM, Boycott BB (1996) The cone synapses of DB1 diffuse, DB6 diffuse and invaginating midget, bipolar cells of a primate retina. J Neurocytol 25:381-390.

Hore PJ, Mouritsen H (2016) The radical-pair mechanism of magnetoreception. Annu Rev Biophys 45:299-344.

Hornstein EP, Verweij J, Li PH, Schnapf JL (2005) Gap-junctional coupling and absolute sensitivity of photoreceptors in macaque retina. J Neurosci 25:11201-11209.

Horváth G (2014) Polarized light and polarization vision in animal sciences, Ed 2. Berlin: Springer.

Hua Y, Laserstein P, Helmstaedter M (2015) Large-volume en-bloc staining for electron microscopy-based connectomics. Nat Commun 6:7923.

Jones CD, Osorio D (2004) Discrimination of oriented visual textures by poultry chicks. Vision Res 44:83-89.

Kihara AH, Paschon V, Cardoso CM, Higa GSV, Castro LM, Hamassaki DE, Britto LRG (2009) Connexin36, an essential element in the rod pathway, is highly expressed in the essentially rodless retina of Gallus gallus. J Comp Neurol 512:651-663.

Kishkinev D, Chernetsov N, Pakhomov A, Heyers D, Mouritsen H (2015) Eurasian reed warblers compensate for virtual magnetic displacement. Curr Biol 25:R822-R824.

Kolb H (1977) The organization of the outer plexiform layer in the retina of the cat: electron microscopic observations. J Neurocytol 6:131-153.

Kolb H, Jones J (1985) Electron microscopy of Golgi-impregnated photoreceptors reveals connections between red and green cones in the turtle retina. J Neurophysiol 54:304-317.

Kremer JR, Mastronarde DN, McIntosh JR (1996) Computer visualization of three-dimensional image data using IMOD. J Struct Biol 116:71-76.

Ladman AJ (1958) The fine structure of the rod-bipolar cell synapse in the retina of the albino rat. J Biophys Biochem Cytol 4:459-466.

Lagnado L, Schmitz F (2015) Ribbon synapses and visual processing in the retina. Annu Rev Vis Sci 1:235-262.

Li H, Chuang AZ, Brien JO (2009) Photoreceptor coupling is controlled by connexin 35 phosphorylation in zebrafish retina. J Neurosci 29:1517815186.

Li W, DeVries SH (2006) Bipolar cell pathways for color and luminance vision in a dichromatic mammalian retina. Nat Neurosci 9:669-675.

Li YN, Tsujimura T, Kawamura S, Dowling JE (2012) Bipolar cell-photoreceptor connectivity in the zebrafish (Danio rerio) retina. J Comp Neurol 520:3786-3802.

Lind O, Kelber A (2011) The spatial tuning of achromatic and chromatic vision in budgerigars. J Vis 11(7):2, 1-9.

Lind O, Chavez J, Kelber A (2014) The contribution of single and double cones to spectral sensitivity in budgerigars during changing light conditions. J Comp Physiol A Neuroethol Sens Neural Behav Physiol 200:197207.

López-López R, López-Gallardo M, Pérez-Alvarez MJ, Prada C (2008) Isolation of chick retina cones and study of their diversity based on oil droplet colour and nucleus position. Cell Tissue Res 332:13-24.

MacNeil MA, Gaul PA (2008) The biocytin wide-field bipolar cell in the rabbit retina selectively contacts blue cones. J Comp Neurol 506: 6-15.

Mariani AP (1984) Bipolar cells in monkey retina selective for the cones likely to be blue-sensitive. Nature 308:184-186

Mariani AP (1987) Neuronal and synaptic organization of the outer plexiform layer of the pigeon retina. Am J Anat 179:25-39.
Mariani AP, Lasansky A (1984) Chemical synapses between turtle photoreceptors. Brain Res 310:351-354.

Mariani AP, Leure-du Pree AE (1978) Photoreceptors and oil droplet colors in the red area of the pigeon retina. J Comp Neurol 182:821838.

Meller K (1964) Elektronenmikroskopische befunde zur differenzierung der photorezeptorzellen und bipolarzellen der retina und ihrer synaptischen verbindungen. Z Zellforch Microsk Anat Histochem 64:733-750.

Mey J, Thanos S (2000) Development of the visual system of the chick: I. Cell differentiation and histogenesis. Brain Res Reviews 32:343-379.

Meyer DB, May HCJ (1973) The topographical distribution of rods and cones in the adult chicken retina. Exp Eye Res 17:347-355.

Millar TJ, Ishimoto I, Chubb IW, Epstein ML, Johnson CD, Morgan IG (1987) Cholinergic amacrine cells of the chicken retina: a light and electron microscope immunocytochemical study. Neuroscience 21:725-743.

Mouritsen H (2018) Long-distance navigation and magnetoreception in migratory animals. Nature 558:50-59.

Naito J, Chen Y (2004) Morphologic analysis and classification of ganglion cells of the chick retina by intracellular injection of lucifer yellow and retrograde labeling with DiI. J Comp Neurol 469:360376.

Nishimura Y, Smith RL, Shimai K (1981) Junction-like structure appearing at apposing membranes in the double cone of chick retina. Cell Tissue Res 218:113-116.

Noel NCL, Allison WT (2018) Connectivity of cone photoreceptor telodendria in the zebrafish retina. J Comp Neurol 526:609-625.

O’Brien JJ, Chen X, Macleish PR, O'Brien J, Massey SC (2012) Photoreceptor coupling mediated by connexin 36 in the primate retina. J Neurosci 32:4675-4687.

Osorio D, Vorobyev M (2005) Photoreceptor spectral sensitivities in terrestrial animals: adaptations for luminance and colour vision. Proc Biol Sci 272:1745-1752.

Owen WG (1985) Chemical and electrical synapses between photoreceptors in the retina of the turtle, Chelydra serpentina. J Comp Neurol 240:423433.

Peddie CJ, Collinson LM (2014) Exploring the third dimension: volume electron microscopy comes of age. Micron 61:9-19.

Pedler CMH, Boyle M (1969) Multiple oil droplets in the photoreceptors of the pigeon. Vision Res 9:525-528.

Polyak SL (1941) The retina: the anatomy and the histology of the retina in man, ape, and monkey, including the consideration of visual functions, the history of physiological optics, and the histological laboratory technique. Chicago: University of Chicago.

Puller C, Haverkamp S, Grünert U (2007) OFF midget bipolar cells in the retina of the marmoset, Callithrix jacchus, express AMPA receptors. J Comp Neurol 502:442-454.

Quesada A, Prada FA, Genis-Galvez JM (1988) Bipolar cells in the chicken retina. J Morphol 197:337-351.

Ribelayga C, Cao Y, Mangel SC (2008) The circadian clock in teh retina controls rod-cone coupling. Neuron 59:790-801.

Ritchey ER, Bongini RE, Code KA, Zelinka C, Petersen-Jones S, Fischer AJ (2010) The pattern of expression of guanine nucleotide-binding protein $\beta 3$ in the retina is conserved across vertebrate species. Neuroscience 169:1376-1391.

Saito T, Kujiraoka T, Yonaha T, Chino J (1985) Reexamination of photoreceptor-bipolar connectivity patterns in carp retina: HRP-EM and GolgiEM studies. J Comp Neurol 236:141-160.

Sakai H, Naka K-I (1983) Synaptic organization involving receptor, horizontal and ON- and OFF-center bipolar cells in the catfish retina. Vision Res 23:339-351.

Schindelin J, Arganda-Carreras I, Frise E, Kaynig V, Longair M, Pietzsch T, Preibisch S, Rueden C, Saalfeld S, Schmid B, Tinevez JY, White DJ, Hartenstein V, Eliceiri K, Tomancak P, Cardona A (2012) Fiji: an open-source platform for biological-image analysis. Nat Methods 9:676-682.

Seifert M, Baden T, Osorio D (2020) The retinal basis of vision in chicken. Semin Cell Dev Biol 106:106-115.

Stavenga DG, Wilts BD (2014) Oil droplets of bird eyes: microlenses acting as spectral filters. Philos Trans R Soc Lond B Biol Sci 369:20130041 
Tanabe K, Takahashi Y, Sato Y, Kawakami K, Takeichi M, Nakagawa S (2006) Cadherin is required for dendritic morphogenesis and synaptic terminal organization of retinal horizontal cells. Development 133:40854096.

Tsukamoto Y, Omi N (2015) OFF bipolar cells in macaque retina: type-specific connectivity in the outer and inner synaptic layers. Front Neuroanat 9:122.

Tsukamoto Y, Omi N (2017) Classification of mouse retinal bipolar cells: type-specific connectivity with special reference to rod-driven aii amacrine pathways. Front Neuroanat 11:92-25.

Waldvogel JA (1990) The bird's eye view. Am Sci 78:342-353.

Walls GL (1942) The vertebrate eye and its adaptive radiation. New York: Hafner.

Wässle H, Puller C, Müller F, Haverkamp S (2009) Cone contacts, mosaics, and territories of bipolar cells in the mouse retina. J Neurosci 29:106-117.

Wiltschko R, Wiltschko W (1995) Magnetic orientation in animals. Berlin, Heidelberg: Springer.

Wood AC (1917) The fundus occuli of birds especially as viewed by the ophthalmoscope. Cleveland: Lakeside.

Worster S, Mouritsen H, Hore PJ (2017) A light-dependent magnetoreception mechanism insensitive to light intensity and polarization. J R Soc Interface 14:20170405.
Xu J, Jarocha LE, Zollitsch T, Konowalczyk M, Henbest KB, Richert S, Golesworthy MJ, Schmidt J, Déjean V, Sowood DJC, Bassetto M, Luo J, Walton JR, Fleming J, Wei Y, Pitcher TL, Moise G, Herrmann M, Yin H, Wu H, Bartölke R, Käsehagen SJ, Horst S, Dautaj G, Murton PDF, Gehrckens AS, Chelliah Y, Takahashi JS, Koch K-W, Weber S, Solov'yov IA, Xie C, Mackenzie SR, Timmel CR, Mouritsen H, Hore PJ (2021) Magnetic sensitivity of cryptochrome 4 from a migratory songbird. Nature. In press.

Yamagata M, Yan W, Sanes JR (2021) A cell atlas of the chick retina based on single-cell transcriptomics. eLife 10:e63907.

Yang X-L, Wu SM (1989) Modulation of rod-cone coupling by light. Science 244:352-354.

Zapka M, Heyers D, Hein CM, Engels S, Schneider N-L, Hans J, Weiler S, Dreyer D, Kishkinev D, Wild JM, Mouritsen H (2009) Visual but not trigeminal mediation of magnetic compass information in a migratory bird. Nature 461:1274-1278

Zoltowski BD, Chelliah Y, Wickramaratne A, Jarocha L, Karki N, Xu W, Mouritsen H, Hore PJ, Hibbs RE, Green CB, Takahashi JS (2019) Chemical and structural analysis of a photoactive vertebrate cryptochrome from pigeon. Proc Natl Acad Sci U S A 116:19449-19457. 